\title{
Public Information Circular
}

for Shipments of Irradiated

Reactor Fuel

\section{U.S. Nuclear Regulatory Commission}

Office of Nuclear Material Safety and Safeguards 


\section{AVAILABILITY NOTICE}

\section{Availability of Reference Materials Cited in NRC Publications}

Most documents cited in NRC publications will be available from one of the following sources:

1. The NRC Public Document Room, 2120 L Street, NW., Lower Level, Washington, DC 20555

2. The Superintendent of Documents, U.S. Government Printing Office, P.O. Box 37082 , Washington, DC 20013-7082

3. The National Technical Information Service, Springfield, VA 22161

Although the listing that follows represents the majority of documents cited in NRC publications, it is not intended to be exhaustive.

Referenced doçuments available for inspection and copying for a fee from the NRC Public Document Room include NRC correspondence and internal NRC memoranda; NRC bulletins, circulars, information notices, inspection and investigation notices; licensee event reports; vendor reports and correspondence; Commission papers; and applicant and licensee documents and correspondence.

The following documents in the NUREG series are available for purchase from the GPO Sales Program: formal NRC staff and contractor reports, NRC-sponsored conference proceedings, international agreement reports, grant publications, and NRC booklets and brochures. Also available are regulatory guides, NRC regulations in the Code of Federal Regulations, and Nuclear Regulatory Commission Issuances.

Documents available from the National Technical Information Service include NUREG-series reports and technical reports prepared by other Federal agencies and reports prepared by the Atomic Energy Commission, forerunner agency to the Nuclear Regulatory Commission.

Documents available from public and special technical libraries include all open literature items, such as books, journal articles, and transactions. Federal Register notices, Federal and State legislation, and congressional reports can usually be obtained from these libraries.

Documents such as theses, dissertations, foreign reports and translations, and non-NRC conference proceedings are available for purchase from the organization sponsoring the publication cited.

Single copies of NRC draft reports are available free, to the extent of supply, upon written request to the Office of Administration, Distribution and Mail Services Section, U.S. Nuclear Regulatory Commission, Washington, DC 20555.

Copies of industry codes and standards used in a substantive manner in the NRC regulatory process are maintained at the NRC Library, 7920 Norfolk Avenue, Bethesda, Maryland, for use by the public. Codes and standards are usually copyrighted and may be purchased from the originating organization or, if they are American National Standards, from the American National Standards Institute, 1430 Broadway, New York, NY 10018. 


\section{DISCLAIMER}

This report was prepared as an account of work sponsored by an agency of the United States Government. Neither the United States Government nor any agency Thereof, nor any of their employees, makes any warranty, express or implied, or assumes any legal liability or responsibility for the accuracy, completeness, or usefulness of any information, apparatus, product, or process disclosed, or represents that its use would not infringe privately owned rights. Reference herein to any specific commercial product, process, or service by trade name, trademark, manufacturer, or otherwise does not necessarily constitute or imply its endorsement, recommendation, or favoring by the United States Government or any agency thereof. The views and opinions of authors expressed herein do not necessarily state or reflect those of the United States Government or any agency thereof. 


\section{DISCLAIMER}

Portions of this document may be illegible in electronic image products. Images are produced from the best available original document. 


\section{Public Information Circular for Shipments of Irradiated Reactor Fuel}

Manuscript Completed: May 1992

Date Published: June 1992

Division of Safeguards and Transportation Office of Nuclear Material Safety and Safeguards U.S. Nuclear Regulatory Commission

Washington, DC 20555

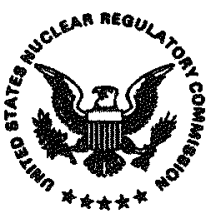




\section{DISCLAIMER}

This report was prepared as an account of work sponsored by an agency of the United States Government. Neither the United States Government nor any agency Thereof, nor any of their employees, makes any warranty, express or implied, or assumes any legal liability or responsibility for the accuracy, completeness, or usefulness of any information, apparatus, product, or process disclosed, or represents that its use would not infringe privately owned rights. Reference herein to any specific commercial product, process, or service by trade name, trademark, manufacturer, or otherwise does not necessarily constitute or imply its endorsement, recommendation, or favoring by the United States Government or any agency thereof. The views and opinions of authors expressed herein do not necessarily state or reflect those of the United States Government or any agency thereof. 


\section{DISCLAIMER}

Portions of this document may be illegible in electronic image products. Images are produced from the best available original document. 



\section{PREFACE}

This circular has been prepared to provide information on the shipment of irradiated reactor fuel (spent fuel) subject to regulation by the U.S. Nuclear Regulatory Commission (NRC). It provides a brief description of spent fuel shipment safety and safeguards requirements of general interest, a summary of data for 1979-1991 highway and railway shipments, and a listing, by State, of recent highway and railway shipment routes.

The enclosed route information reflects specific NRC approvals that have been granted in response to requests for shipments of spent fuel. This publication does not constitute authority for carriers or other persons to use the routes described to ship spent fuel, other categories of nuclear waste, or other materials. 


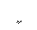




\section{CONTENTS}

Page

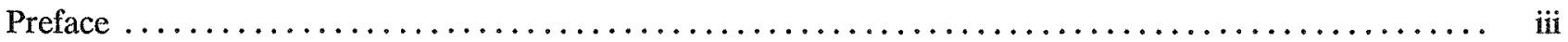

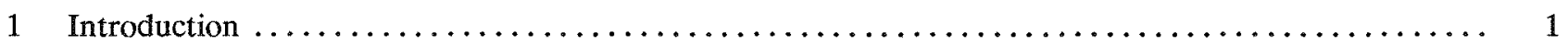

2 Regulatory Requirements for Spent Fuel Shipments $\ldots \ldots \ldots \ldots \ldots \ldots \ldots \ldots \ldots \ldots \ldots \ldots \ldots \ldots$

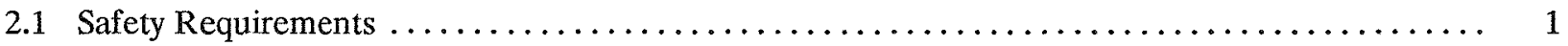

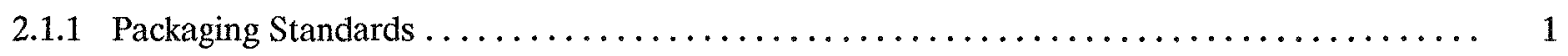

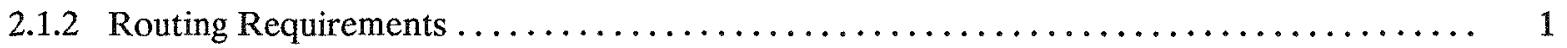

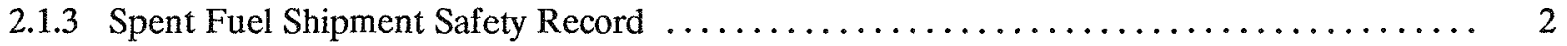

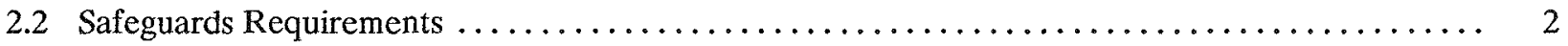

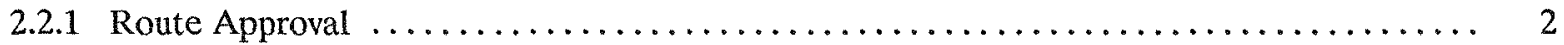

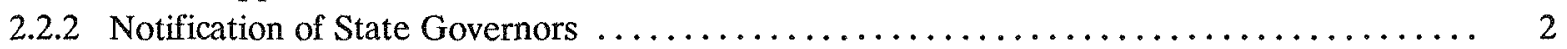

2.2.3 Spent Fuel Shipment Safeguards Record $\ldots \ldots \ldots \ldots \ldots \ldots \ldots \ldots \ldots \ldots \ldots \ldots \ldots \ldots \ldots$

3 Descriptive Statistics for 1979-1991 Highway and Railway Spent Fuel Shipments .............. 3

4 Route Segment Listing for Recent Highway and Railway Spent Fuel Shipments ................ 4

\section{FIGURES}

3.1 Routes Used for Spent Fuel Shipments: $1979-1991 \ldots \ldots \ldots \ldots \ldots \ldots \ldots \ldots \ldots \ldots \ldots \ldots \ldots \ldots$

3.2 Number of Spent Fuel Shipments by Mode: $1979-1991 \ldots \ldots \ldots \ldots \ldots \ldots \ldots \ldots \ldots \ldots \ldots \ldots$

3.3 Number of Spent Fuel Shipments by Year: $1979-1991 \ldots \ldots \ldots \ldots \ldots \ldots \ldots \ldots \ldots \ldots \ldots \ldots \ldots . . . \ldots$

3.4 Quantity of Spent Fuel Shipped by Mode: $1979-1991 \ldots \ldots \ldots \ldots \ldots \ldots \ldots \ldots \ldots \ldots \ldots \ldots \ldots \ldots$

3.5 Quantity of Spent Fuel Shipped by Year: $1979-1991 \ldots \ldots \ldots \ldots \ldots \ldots \ldots \ldots \ldots \ldots \ldots \ldots \ldots$

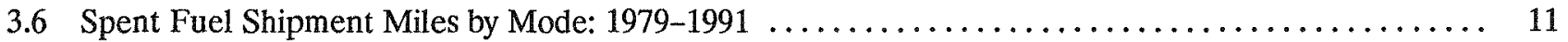

3.7 Spent Fuel Shipment Miles by Year: $1979-1991 \ldots \ldots \ldots \ldots \ldots \ldots \ldots \ldots \ldots \ldots \ldots \ldots \ldots \ldots \ldots$

3.8 Spent Fuel Shipment Kilogram-Miles by Mode: $1979-1991 \ldots \ldots \ldots \ldots \ldots \ldots \ldots \ldots \ldots \ldots \ldots \ldots . \ldots 12$

3.9 Spent Fuel Shipment Kilogram-Miles by Year: $1979-1991 \ldots \ldots \ldots \ldots \ldots \ldots \ldots \ldots \ldots \ldots \ldots \ldots$

3.10 Number and Total Quantity of Spent Fuel Shipments by Shipment Quantity Range: $1979-1991$. . .. 13

3.11 Number and Total Quantity of Spent Fuel Shipments by Shipment Distance Range: 1979-1991 . . . . 14

\section{TABLES}

3.1 Number of Shipments and Quantity of Spent Fuel Shipped for Origination/Destination

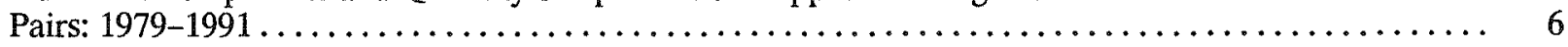

3.2 Summary Data for 1979-1991 Spent Fuel Shipment Information . . . . . . . . . . . . . . . 7

3.3 Number of Domestic and International Spent Fuel Shipments: $1979-1991 \ldots \ldots \ldots \ldots \ldots \ldots . . . .6$

4.1 Highway and Railway Spent Fuel Shipment Routes Used in $1987-1991$. . . . . . . . . . . . 15 


\section{INTRODUCTION}

The Nuclear Regulatory Commission (NRC) is authorized under the Atomic Energy Act of 1954, as amended, to regulate the commercial nuclear industry for the purpose of protecting the public health and safety and the common defense and security of the United States. Included in this authority is the regulation of certain aspects of the transportation of irradiated reactor fuel (spent fuel). NRC's role in this regard is explained in Section 2 of this report. Section 3 provides descriptive statistics on spent fuel shipments in the commercial nuclear industry for the period 1979-1991. Section 4 contains a listing, by State, of highway and railway segments used in each State for transporting spent fuel in recent years (1987-1991).

\section{REGULATORY REQUIREMENTS FOR SPENT FUEL SHIPMENTS}

Spent fuel shipments are regulated from both the safety and safeguards standpoints. Safety deals with protection of public health and safety during routine transport as well as in the event of handling or transportation accidents, whereas safeguards deals with the protection of shipments against deliberate, malevolent acts by persons.

\subsection{Safety Requirements}

Federal regulatory responsibility for spent fuel transportation safety is shared by NRC and the U.S. Department of Transportation (DOT). Basically, NRC's safety role is to ensure that the spent fuel packagings meet strict regulatory design rules, and includes approving packaging designs and Quality Assurance Programs, and conducting inspections. NRC packaging requirements are specified in 10 CFR Part 71. The DOT role in regulating spent fuel shipment safety is broad, and covers all aspects of actual transportation, including route selection, vehicle condition and placarding, driver training, package marking, labeling, other shipping documentation, efc. These requirements are specified in 49 CFR Parts 171-178. Of the NRC and DOT safety requirements for spent fuel shipments, NRC's packaging standards and DOT's routing rules have been of most general interest, and are briefly described below.

\subsubsection{Packaging Standards}

The basic strategy for regulating spent fuel shipments is to rely on the packaging to protect the public health and safety during transportation. The packaging standards that have been established in the regulations provide that a spent fuel packaging (cask) shall prevent the loss or dispersion of the radioactive contents, provide adequate shielding and heat dissipation, and prevent nuclear criticality under both normal and accident conditions of transportation. The normal conditions of transportation that must be considered are specified in the regulations in terms of hot and cold environments, pressure differential, vibration, water spray, impact, puncture, and compression tests. Accident conditions that must be considered are specified in terms of impact, puncture, fire conditions, and immersion.

The NRC initially reviews the cask design to verify its resistance to accidents. NRC must issue a certificate before a cask fabricated from the reviewed design can be used to transport spent fuel.

The ability of packaging to provide protection has been demonstrated by the responses of packaging during actual traffic accidents. For example, an accident occurred on December 8, 1971, on a major highway near Oak Ridge, Tennessee. In this accident, the driver of a vehicle carrying a spent fuel cask swerved to avoid colliding with an oncoming vehicle, lost control, and overturned off the roadway. The cask assembly was thrown into a ditch, traveling more than 100 feet before coming to rest. No release of contents or release of radiation occurred. The outer surface of the cask substained minor damage. The spent fuel cask was placed on another trailer and taken to its destination. The cask was returned to service after repair of the minor damage and inspection.

The accident resistance of casks has also been demonstrated in controlled tests. In one test, sponsored by the U.S. Department of Energy (DOE), a truck bearing a cask was deliberately placed in the path of and struck by a 120-ton locomotive traveling about 80 miles per hour. In another DOE test, a cask aboard a truck moving about 80 miles per hour was deliberately crashed into an immovable concrete structure. Subsequent examination in both these tests confirmed that no radioactive material would have been released from the casks had they been loaded with spent fuel. Thus, both field experience and controlled tests have substantiated the strategy of depending on packaging design for safety in transit.

For further information on spent fuel shipment safety, please consult NUREG/BR-0111, Transporting Spent Fuel-Protection Provided Against Severe Highway and Railroad Accidents."

\subsubsection{Routing Requirements}

DOT requirements in 49 CFR 177.825 (b) designate the use of the Interstate System of highways and available city beltways as the primary roadways over which radioactive material shipments under an NRC safeguards-approved route plan are to be carried. There is no routing rule for rail shipments. The general designation as preferred 
highways is given to roadways, based on their capacity for reducing transit times. Appropriate State routing agencies, following prescribed criteria, may designate an alternate route to the preferred Interstate System. It is the responsibility of spent fuel carriers to abide by the routing rule when they transport spent fuel by highway.

\subsubsection{Spent Fuel Shipment Safety Record}

The safety record for spent fuel shipments in the U.S. and in other industrialized nations is enviable. Of the thousands of shipments completed over the last 30 years, none has resulted in an identifiable injury through release of radioactive material.

\subsection{Safeguards Requirements}

In May 1979, NRC adopted new regulations, in $10 \mathrm{CFR}$ 73.37, for strengthening the protection of shipments of spent fuel against radiological sabotage. These regulations were subsequently revised in May 1980, in response to public comments. The regulations require, among other actions, NRC approval of routes for the transportation of spent fuel, to ensure adequate planning for physical protection against actual or attempted acts of radiological sabotage. Physical protection requirements for NRC licensees who transport or deliver spent fuel to a carrier for transport include: shipment prenotification to NRC; procedures for coping with emergencies; a communications center; contact with the communications center every 2 hours; a written log of shipment events; arrangements with local law enforcement agencies (NRC often coordinates this item); avoidance of intermediate stops; surveillance of the shipment vehicle while stopped; armed escorts in heavily populated areas; escort training; onboard communications; immobilization devices on trucks; driver training; and notification of State governors before shipments. Of these safeguards requirements, route approval and State notification have been of most general interest, and are briefly described below.

\subsubsection{Route Approval}

NRC licensees planning to ship spent fuel are required to submit proposed routes for such shipments to the NRC for approval, from the safeguards standpoint, before the use of a given route. For highway shipments, the licensee must propose a route that conforms with DOT's routing rules. NRC surveys proposed routes for communication reception, location of safe havens, etc. Routes may be approved for a single shipment, or a specified series of shipments. Once a shipment series is approved, the route may be used for all shipments, without reapproval of the route for each shipment, provided that NRC is notified in advance of each shipment. The route approval is for a stated number of shipments only; any subsequent shipments not part of an approval must be additionally approved. NRC approval authorizes only spent fuel shipments, and does not include other categories of nuclear waste material. From time to time, NRC may authorize alternate routes or detours, as circumstances dictate at the time of shipment. Also, detours may be taken without prior approval, in response to unforeseen circumstances that arise during a shipment. Criteria for determining when and how such detours may be taken are provided in published regulatory guidance (Physical Protection of Irradiated Spent Fuel,"NUREG-0561, Rev. 1).

\subsubsection{Notification of State Governors}

NRC requires its licensees to notify the governor or the governor's designee before the transport of spent fuel within or through the State [10 CFR 73.37 (f)]. Spent fuel is identified in 10 CFR 73.37(a) as ". . net weight of irradiated fuel, exclusive of cladding or other structural or packaging material. ..." The notification must be in writing and postmarked at least 7 days before transport, if mailed, or delivered at least 4 days before transport, if sent by messenger. The notification must include:

- the name, address, and telephone number of the shipper, carrier, and receiver

- a description of the shipment, as specified by DOT

- a listing of the routes to be used within the State

- a statement that NRC requires that shipment schedule information (provided as an enclosure) be protected from unauthorized disclosure.

The enclosure to the notification provides:

- the estimated date and time of departure from the point of origin of the shipment

- the estimated date and time of entry into the governor's State

- a statement that schedule information must be protected from unauthorized disclosure until at least 10 days after the shipment (or 10 days after the last shipment of a series) has entered or originated within the State.

The licensee must also notify the governor of schedule changes that differ by more than 6 hours from the furnished schedule. Subsequent distribution of the schedule information is at the governor's discretion, but NRC regulations require all persons who receive the schedule information to protect it from unauthorized disclosure. 


\subsubsection{Spent Fuel Shipment Safeguards Record}

Safeguards incidents for spent fuel shipments are those that involve attempts at radiological sabotage of spent fuel, or purposeful acts that threaten or result in significant degradation of the safeguards system used to protect the shipment. Licensees are required, under existing regulations, to immediately notify law enforcement authorities upon the occurrence or discovery of a safeguards incident, for the purpose of initiating an appropriate response. In addition, licensees are required to promptly report safeguards incidents to NRC by telephone, followed by a written report. To date, no safeguards incidents involving the shipment of spent fuel have occurred. However, a number of citations have been issued for minor procedural infractions: one in 1986, two in 1987, two in 1990 and one in 1991.

\section{DESCRIPTIVE STATISTICS FOR 1979-1991 HIGHWAY AND RAILWAY SPENT FUEL SHIPMENTS}

NRC began approving spent fuel shipments in 1979. This section provides descriptive statistics on the shipments that have occurred through 1991. Only shipments of academic, industrial, and utility irradiated reactor fuel subject to NRC regulation are included; DOE shipments are excluded. Figure 3.1 and Table 3.1 provide a geographical perspective for spent fuel shipments. Figure 3.1 shows the highway and railway routes used for spent fuel transport during 1979-1991. Table 3.1 shows the number of shipments and quantity of spent fuel shipped between specific origination/destination pairs for the period.

Table 3.2 and Figures $3.2-3.9$ provide more detailed spent fuel shipment information, including mode of shipment (highway or railway) and shipment trends over time. Table 3.2 provides a summary of spent fuel shipment data for the 1979-1991 period. For each year, the table provides four variables that describe shipping activity by mode. Data for shipment miles are taken primarily from road atlases, and have been rounded to the nearest hundred miles for each year. Data on quantity of spent fuel shipped were provided by shippers, and have been rounded to the nearest hundred kilograms for each year. The kilogram-miles data are derived from shipment quantity and distance data, and have been rounded to the nearest hundred thousand.

Table 3.3 shows the pattern of highway and rail shipments throughout the period 1979-1991. The number of domestic highway shipments (except for the year 1981) rose steadily to a high in 1984 then declined steadily through 1991. Import shipments have generally declined since 1980, with no imports in 1990 and 1991. The number of export shipments has been low (0-3) through the entire period. Also, in 1990-1991 four international shipments have been made in which US ports were transitted.

Figure 3.2 shows that most ( 91.8 percent) of approximately 1,200 spent fuel shipments during the 1979-1991 period were completed over highways. Figure 3.3 shows that most of the shipping activity occurred during 1980-1987, with a generally decreasing trend in the number of shipments since 1984 .

Figure 3.4 shows that the larger quantity ( 64.0 percent) of spent fuel was shipped by railway, which reflects the greater capacity of rail spent fuel containers versus that for trucks. In addition, a few rail shipments included multiple spent fuel containers, further increasing the rail shipment payload. The figure indicates that 989,500 kilograms, or about 990 metric tons, of spent fuel were shipped. Figure 3.5 shows that greater quantities of spent fuel were shipped during 1984-1987, than before or after those years. The figure also shows that 1985 was the peak year for quantity of spent fuel shipped, and that, since then most spent fuel has been shipped by rail.

Figure 3.6 shows that the highway mode accounted for most ( 94.8 percent) of the 774,000 spent fuel shipment miles. Figure 3.7 shows that shipment mileage peaked in 1984 , with a general decline in subsequent years.

Figure 3.8 shows the cumulative movement of spent fuel, calculated by summing the product of quantity and distance for all shipments, and is expressed in kilogrammiles. This unit is analogous to "ton-miles," a unit commonly used to measure the flow of commodities. The figure shows that the railway mode accounted for the majority (64.8 percent) of the 396.1 million kilogram-miles associated with spent fuel shipments. Figure 3.9 shows the kilogram-miles distribution by year.

Finally, Figures 3.10 and 3.11 provide an operational perspective for the spent fuel shipments. Figure 3.10 shows the distribution of shipments by individual shipment quantity, and the corresponding total quantity shipped. The individual shipment quantities have been grouped into ranges, with highway shipments most frequently falling within the $0-10,400-500$, and 1300-1400 kilogram ranges, and with most railway shipments within the $6000-7000$ kilogram range. The smallest quantity range accounts for the largest number of shipments (478, or 39 percent of highway and railway shipments combined), yet comprises only 2,000 kilograms ( 0.2 percent) of the combined quantity shipped. Conversely, the 54 railway shipments in the 6000-7000 kilogram shipment range comprise only 4.5 percent of the combined total shipments, but 36 percent of the combined quantity of spent fuel 
shipped. (All shipments less than 1400 kilograms were shipped by highway, and all shipments greater than 1400 kilograms were shipped by railway.)

Figure 3.11 shows the distribution of shipments by distance range, and the corresponding total quantity of spent fuel shipped. For highway shipments, the number of shipments generally decreases with shipment distance, although a significant number of shipments exceeded 900 miles. Of the 356 metric tonnes shipped by highway, 173 tonnes ( 49 percent) traveled less than 200 miles. The number of rail shipments was somewhat uniform over the ranges, with 400-700 mile shipments accounting for 62 percent of the total quantity shipped by rail.

\section{ROUTE SEGMENT LISTING FOR RECENT HIGHWAY AND RAILWAY SPENT FUEL SHIPMENTS}

Table 4.1 is a listing of highway and railway routes that have recently been used to transport spent fuel. The table identifies the spent fuel shipments that occurred in each State. The table also lists the highway or railway route segments within the State that were used for each shipment, and when the shipment was completed. The table shows that highway spent fuel shipments were made in or through 26 States, and that railway shipments were made in or through 7 States during the period discussed. 


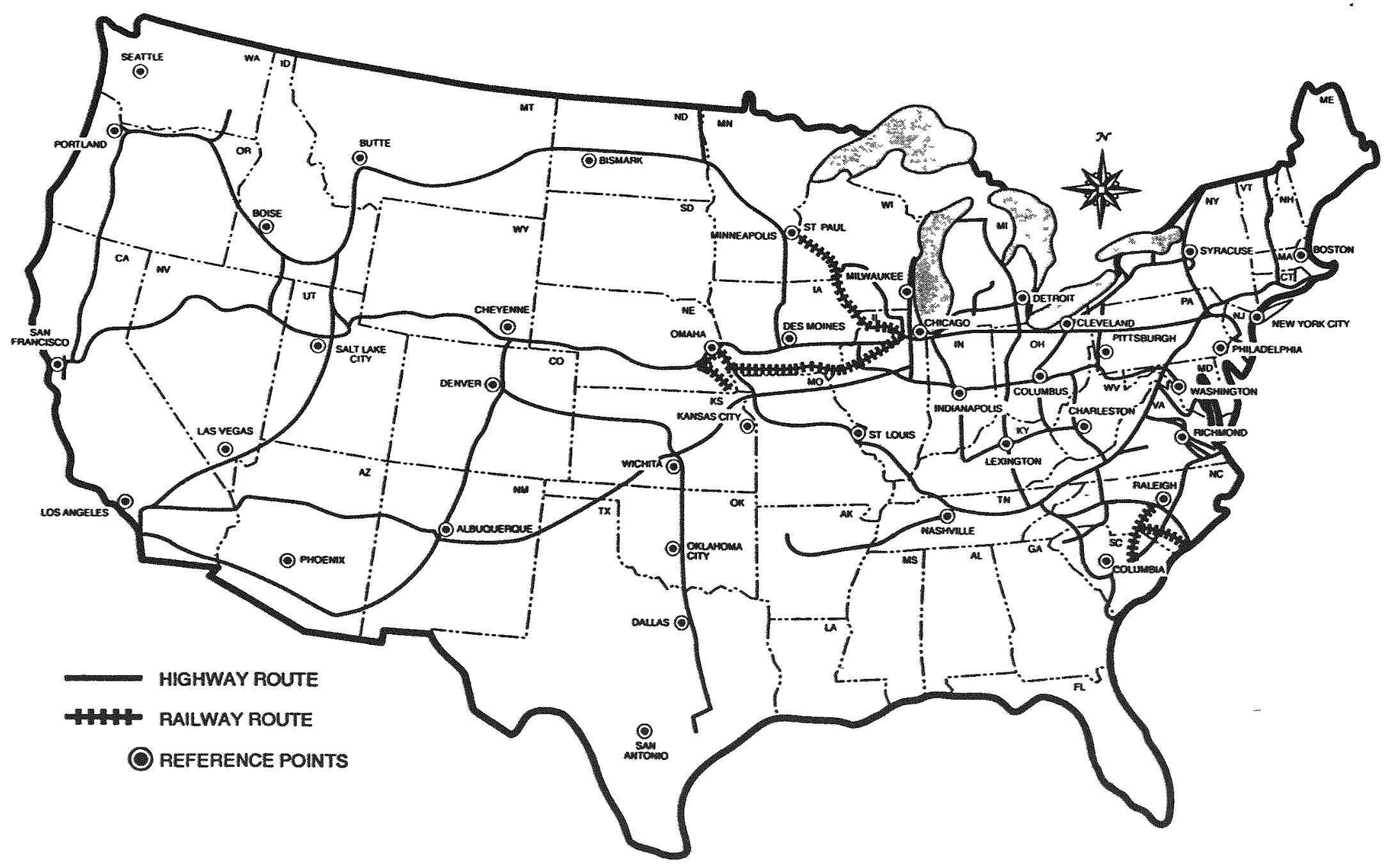


Alexanctia Bay, NY*

Arkansas One, AR

Babcock \& Wilcox, VA

Batelle-columbus, OH

Big Rock Poimt, Mi

\begin{tabular}{l} 
Byron Station, 1 L \\
\hline Brunswick, NC (via railway) \\
\hline
\end{tabular}

Catven Clifits, MD

CINTICHEM. NY

Cooper. NE I via railway

Derby Lane, VT*

Dresden Station.

\begin{tabular}{l} 
Fort Calhoun, $N$. \\
\hline Fon Enie. $N Y^{*}$ \\
\hline
\end{tabular}

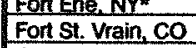

Goneral Atomiics, CA

$a$

\begin{tabular}{l} 
General Atomics, \\
\hline GEMaris IL \\
\hline GENallecitos, CA \\
\hline GEMilmingon
\end{tabular}

GEMilimination, NC

Haddarm Neck, CT

Michigan State Univ

Millstone, CT

Monticello, MN (via railway)

Nat'l. Inst. Sids, \& Tech. MD

\begin{tabular}{l} 
Newport News, VA** \\
\hline Nornolk Int'l Teminal VA**
\end{tabular}

Nuciear Fuel Senvice, West Valley, Ny

Oconee, SC

Oadensbura. NY*

Oyster Creek, NJ

Pembina, ND*

Ponland, OR*

Port of Oakland, CA*

Porolavan ca Gá

Quad Cities, IL

Rnode Island A E C R

Richmond $\mathrm{CA}^{*}$

Robinson SC Nia rainwav):-

San Onofre,

Univ. of Caliomia (Berkeley) CA

Univ. of Michigan, MI

\begin{tabular}{l} 
Univ. Of Micchigan , MI \\
\hline Univ. of Missouri, MO
\end{tabular}

\begin{tabular}{l} 
Univ. of Virginia, VA \\
\hline Virail S Summer SC*
\end{tabular}

Zion, il

* Port of Entry
** Port of Departure

Table 3.2 Summary Data for 1979-1991 Spent Fuel Shipment Information

\begin{tabular}{|c|c|c|c|c|c|c|c|c|}
\hline \multirow{3}{*}{ Year } & \multicolumn{2}{c|}{$\begin{array}{c}\text { Number of } \\
\text { Shipments }\end{array}$} & $\begin{array}{c}\text { Kilograms } \\
\text { Spent Fuel Shipped } \\
\text { (Thousand) }\end{array}$ & \multicolumn{2}{c|}{$\begin{array}{c}\text { Shipment } \\
\text { Miles } \\
\text { (Thousand) }\end{array}$} & \multicolumn{2}{c|}{$\begin{array}{c}\text { Kllogram- } \\
\text { Miles } \\
\text { (Millon) }\end{array}$} \\
\cline { 2 - 9 } & Highway & Railway & Highway & Railway & Highway & Railway & Highway & Railway \\
\hline 1979 & 16 & 11 & 0.1 & 30.2 & 8.0 & 2.3 & 0.1 & 6.2 \\
1980 & 130 & 5 & 10.0 & 13.6 & 115.9 & 1.0 & 17.2 & 2.8 \\
1981 & 81 & 2 & 7.9 & 6.0 & 38.5 & 0.4 & 1.7 & 1.2 \\
1982 & 124 & 0 & 7.1 & 0.0 & 106.8 & 0.0 & 1.8 & 0.0 \\
1983 & 117 & 0 & 36.6 & 0.0 & 83.6 & 0.0 & 12.7 & 0.0 \\
1984 & 245 & 3 & 84.5 & 23.8 & 181.3 & 1.6 & 51.4 & 12.7 \\
1985 & 135 & 18 & 74.0 & 119.4 & 70.9 & 8.7 & 28.3 & 57.8 \\
1986 & 105 & 15 & 40.4 & 97.5 & 47.8 & 8.7 & 8.8 & 56.3 \\
1987 & 107 & 15 & 82.3 & 101.4 & 41.8 & 8.4 & 14.8 & 56.5 \\
1988 & 25 & 7 & 12.8 & 41.8 & 11.4 & 4.3 & 2.4 & 25.7 \\
1989 & 16 & 6 & 0.1 & 30.8 & 16.7 & 1.7 & 0.1 & 8.7 \\
1990 & 2 & 8 & $(0.03)$ & 70.5 & 1.5 & 1.6 & 10.02 * & 13.8 \\
1991 & 11 & 10 & 0.1 & 98.4 & 9.6 & 1.5 & 0.1 & 15.0 \\
\hline TOTAL & 1114 & 100 & 355.9 & 633.5 & 733.8 & 40.2 & 139.4 & 256.7 \\
\hline
\end{tabular}

* Entries in parentheses rounded to nearest hundredth: All others rounded to nearest tenth. 
Table 3.3 Number of Domestic and International Spent Fuel Shipments: 1979-1991

\begin{tabular}{|c|c|c|c|c|c|}
\hline \multirow{3}{*}{ Year } & \multicolumn{2}{|c|}{ Domestlc } & \multicolumn{3}{c|}{ International } \\
\cline { 2 - 6 } & Highway & Railway & Export & Import & Transient \\
\hline 1979 & 2 & 11 & 0 & 14 & 0 \\
1980 & 73 & 5 & 2 & 55 & 0 \\
1981 & 30 & 2 & 3 & 48 & 0 \\
1982 & 80 & 0 & 1 & 43 & 0 \\
1983 & 92 & 0 & 2 & 23 & 0 \\
1984 & 209 & 3 & 2 & 34 & 0 \\
1985 & 114 & 18 & 0 & 21 & 0 \\
1986 & 88 & 15 & 0 & 17 & 0 \\
1987 & 85 & 15 & 3 & 19 & 0 \\
1988 & 10 & 7 & 0 & 15 & 0 \\
1989 & 11 & 6 & 1 & 4 & 0 \\
1990 & 0 & 8 & 2 & 0 & 3 \\
1991 & 7 & 10 & 4 & 0 & 1 \\
\hline
\end{tabular}




\section{TOTAL NUMBER OF SHIPMENTS - 1214}

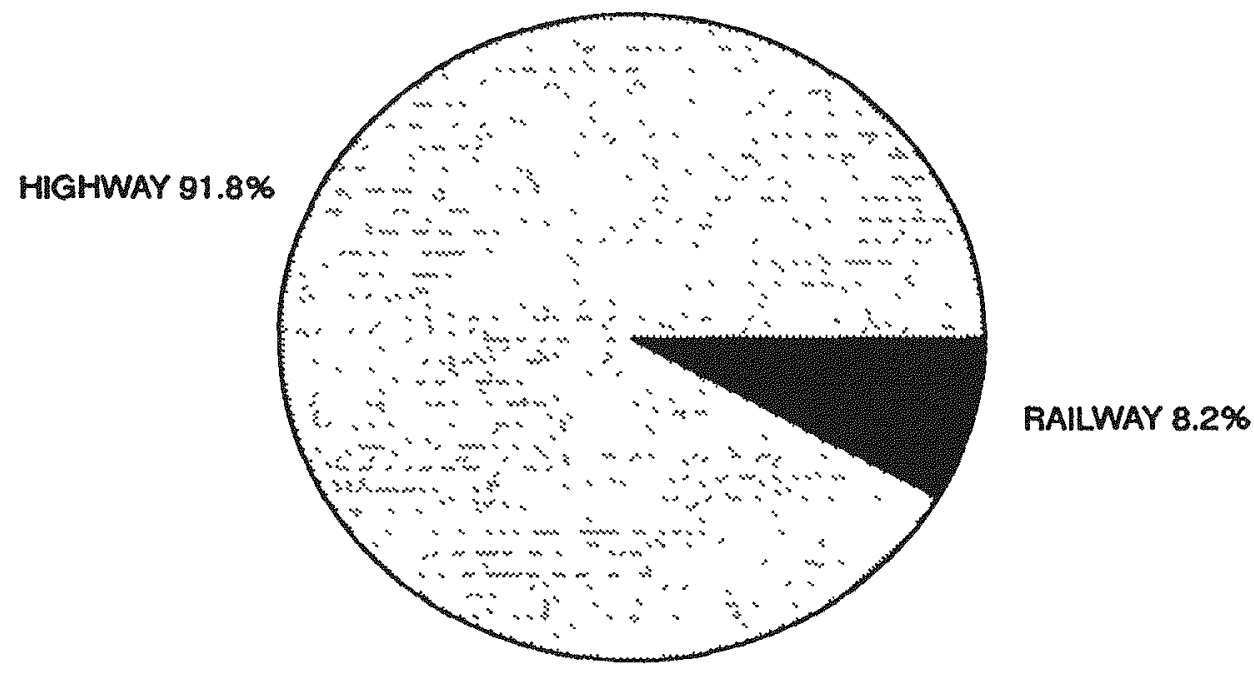

Figure 3.2 Number of Spent Fuel Shipments by Mode: 1979-1991

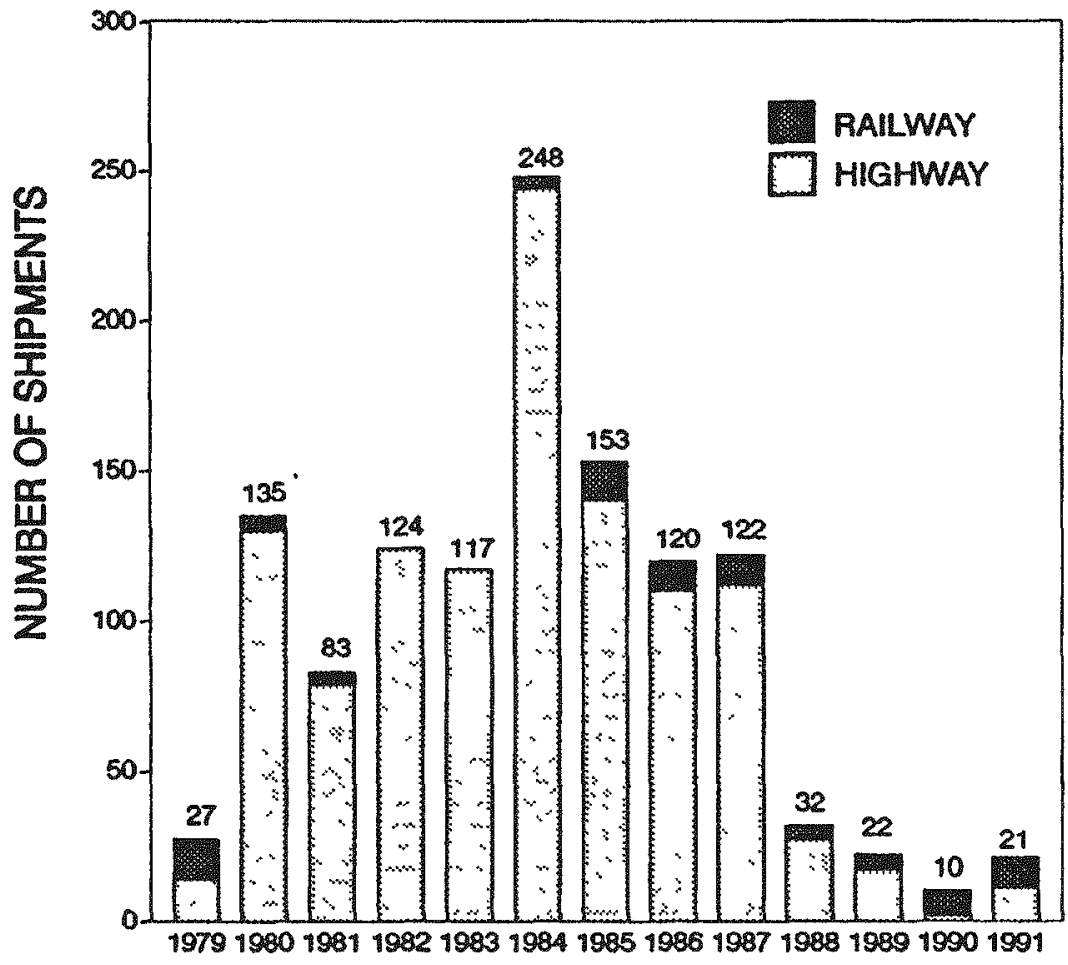

Figure 3.3 Number of Spent Fuel Shipments by Year: 1979-1991 


\section{TOTAL KILOGRAMS OF SPENT FUEL SHIPPED - 989.5 THOUSAND}

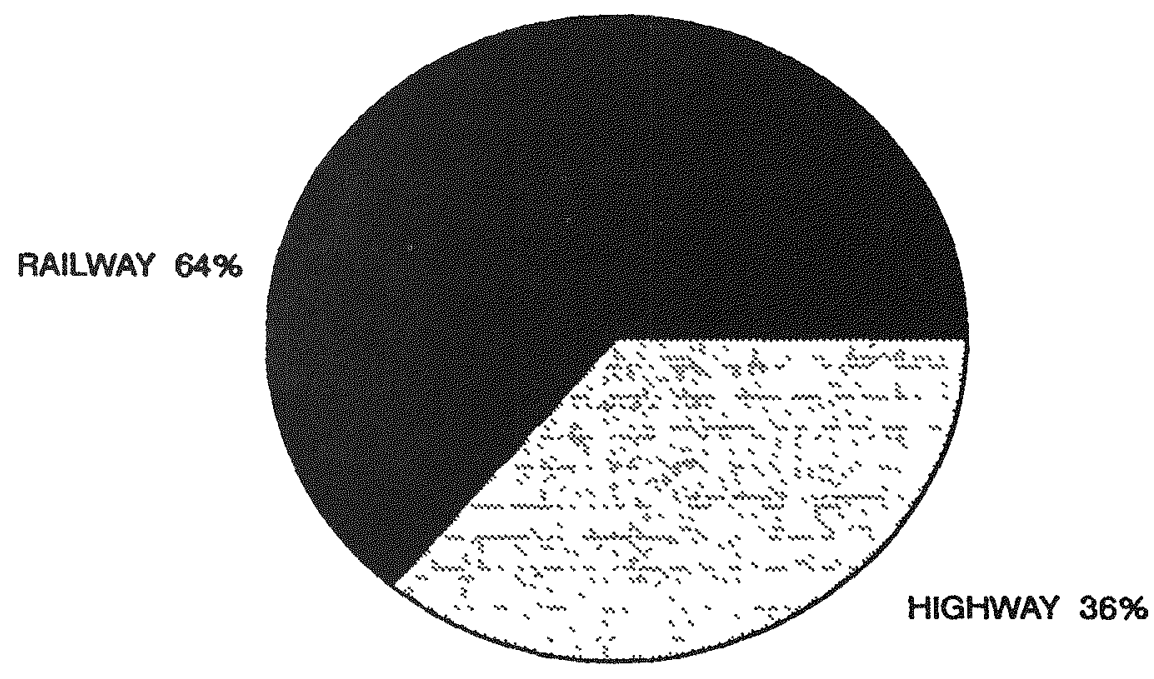

Figure 3.4 Quantity of Spent Fuel Shipped by Mode: 1979-1991

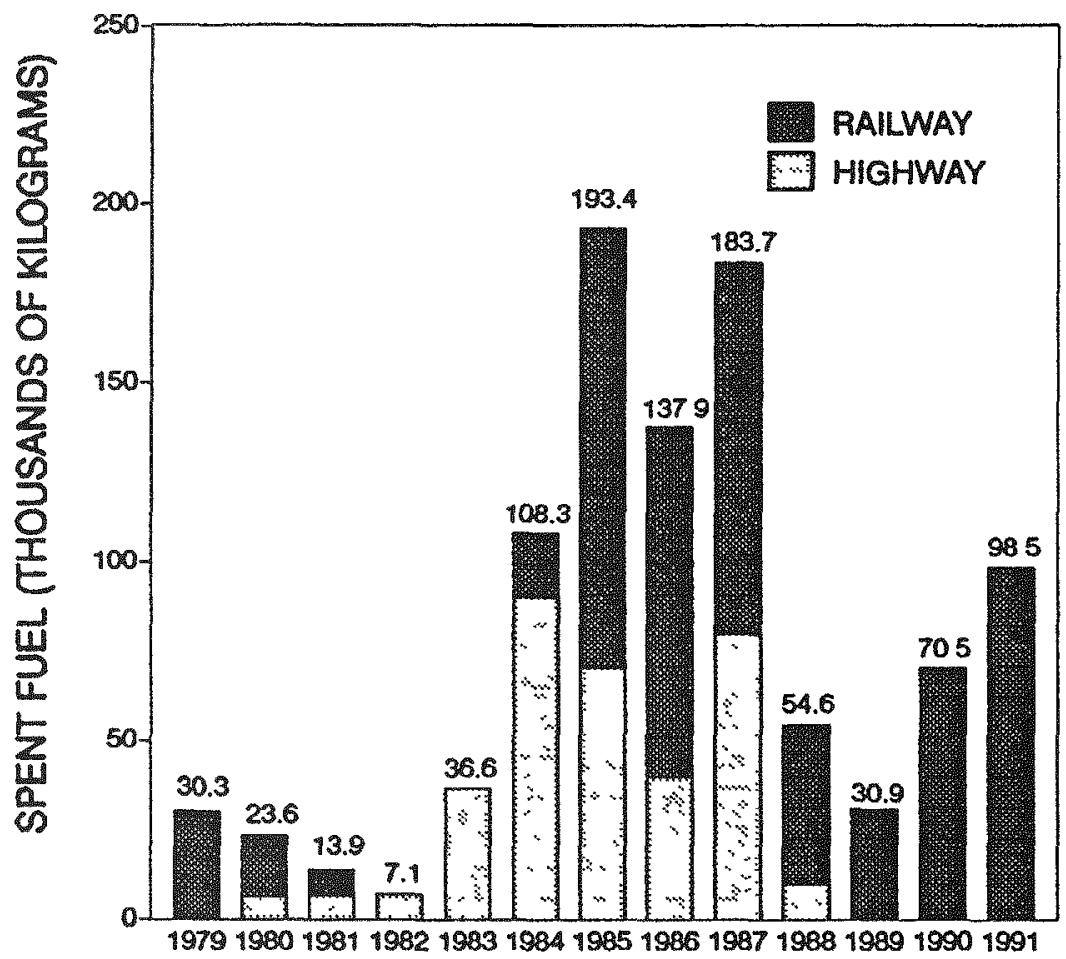

Figure 3.5 Quantity of Spent Fuel Shipped by Year: 1979-1991 
TOTAL SHIPMENT MILES - 774,000

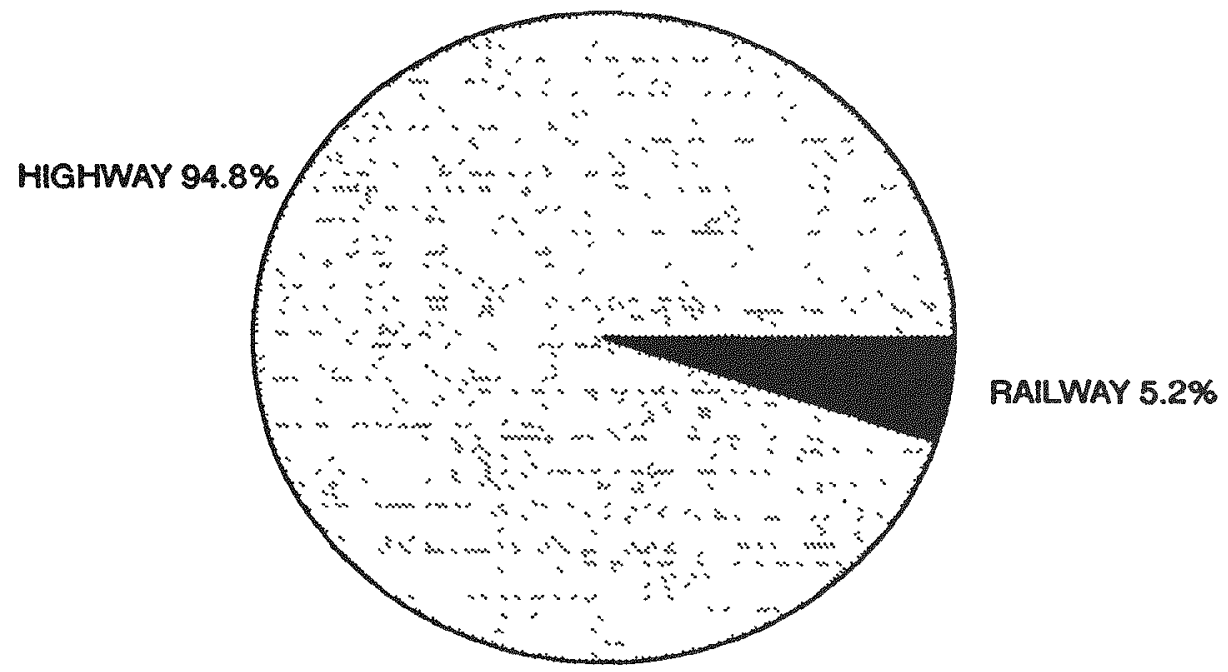

Figure 3.6 Spent Fuel Shipment Miles by Mode: 1979-1991

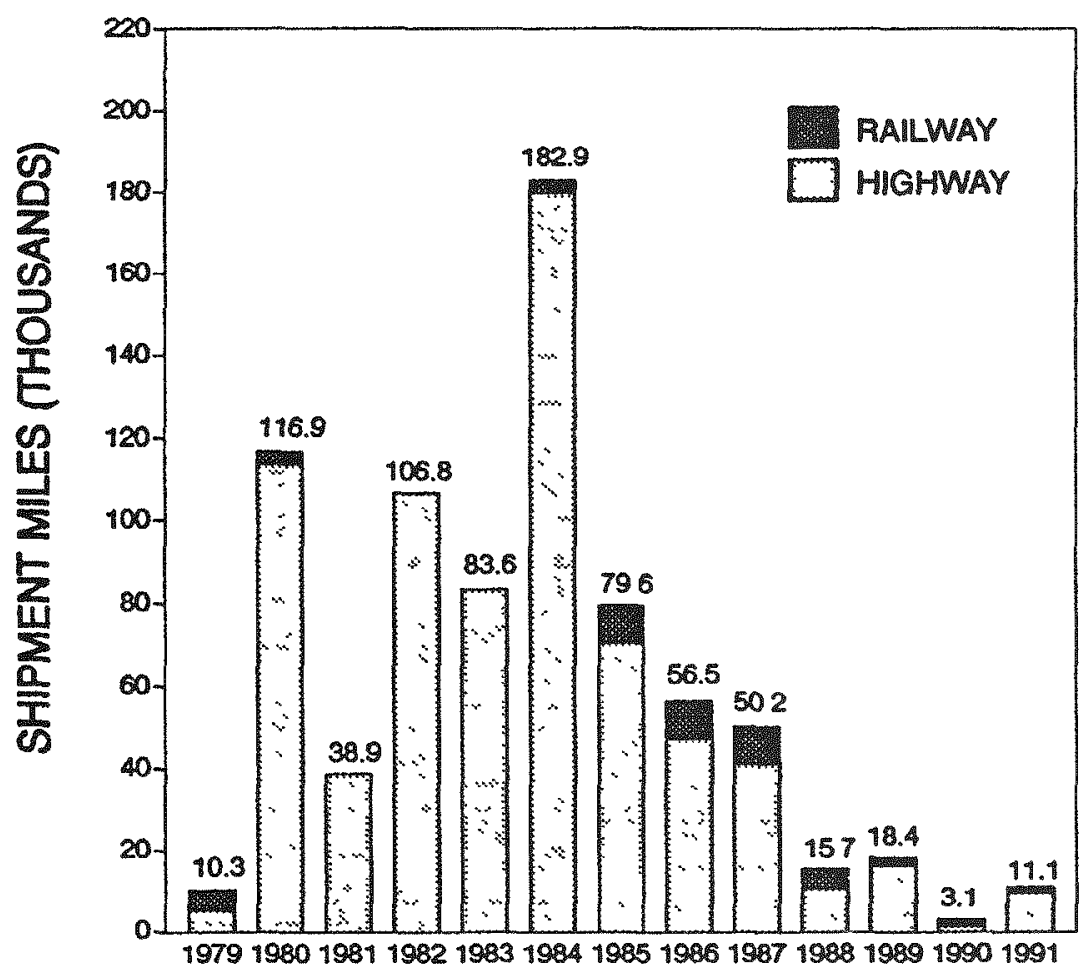

Figure 3.7 Spent Fuel Shipment Miles by Year: 1979-1991 
TOTAL KILOGRAM-MILES - 396.1 MILLION

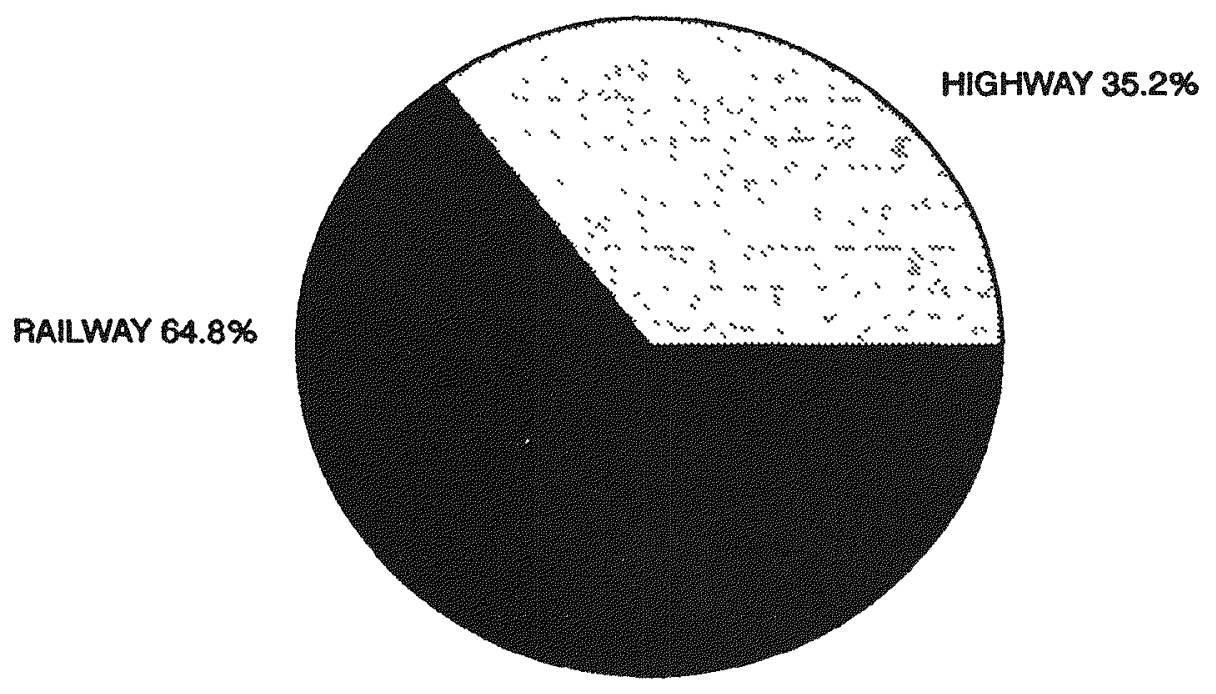

Figure 3.8 Spent Fuel Shipment Kilogram-Miles by Mode: 1979-1991

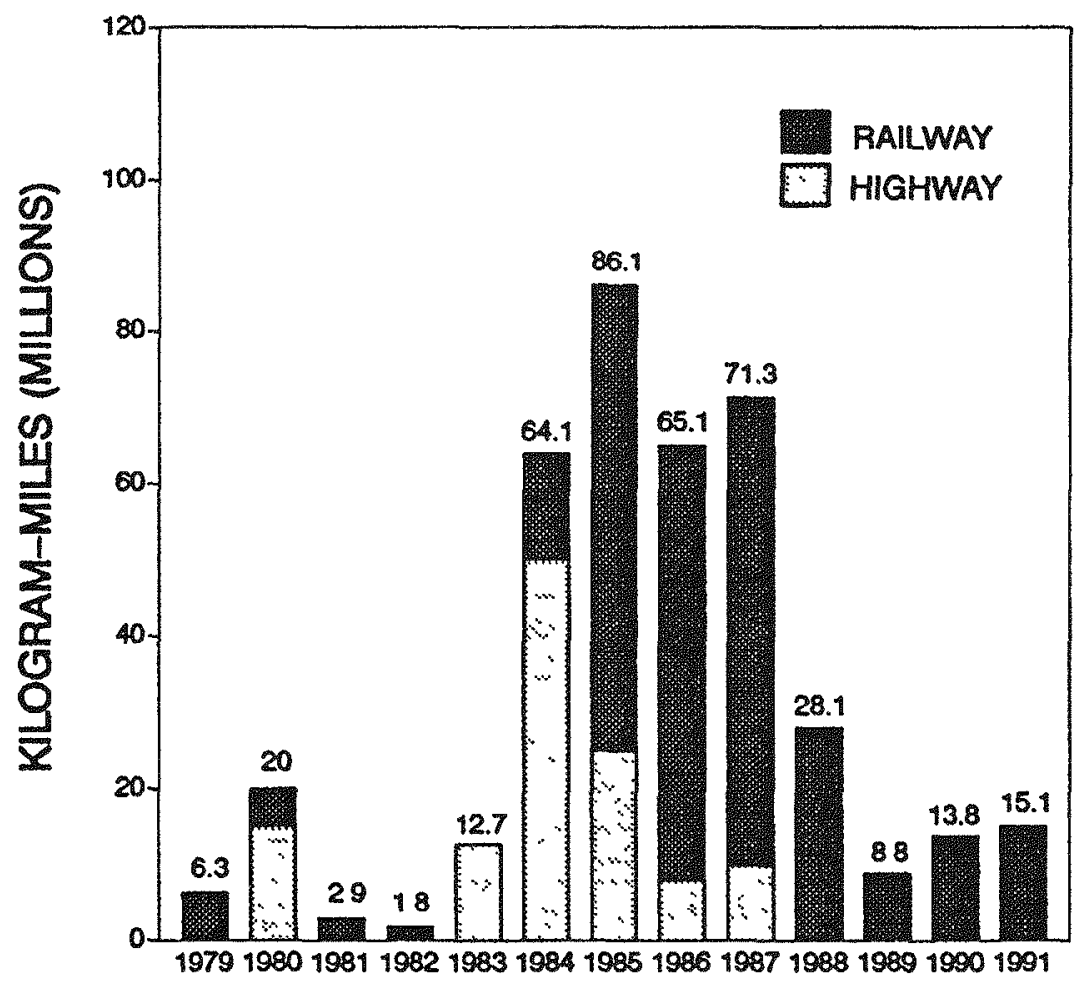

Figure 3.9 Spent Fuel Shipment Kilogram-Miles by Year: 1979-1991 

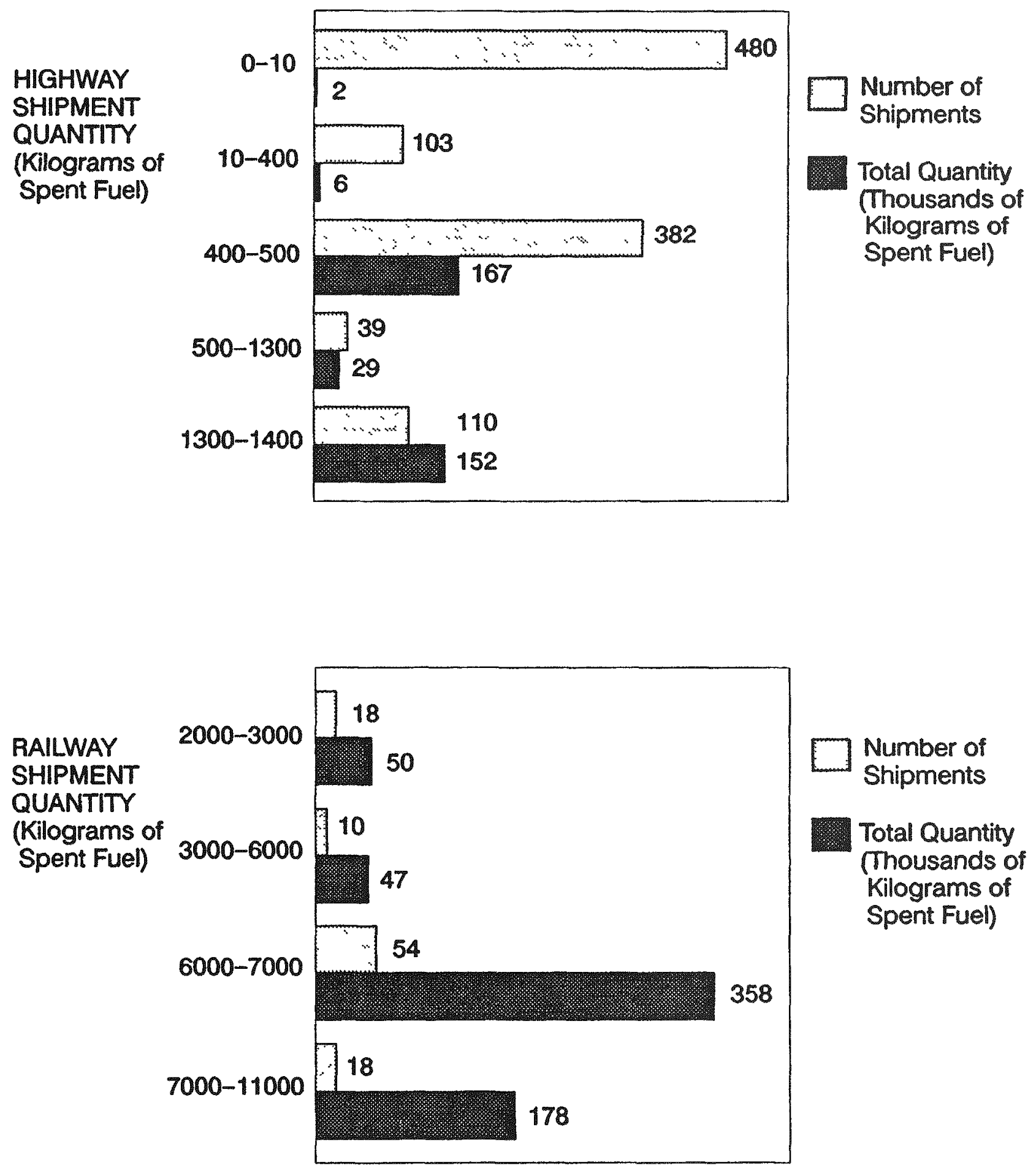

Figure 3.10 Number and Total Quantity of Spent Fuel Shipments by Shipment Quantity Range: 1979-1991 

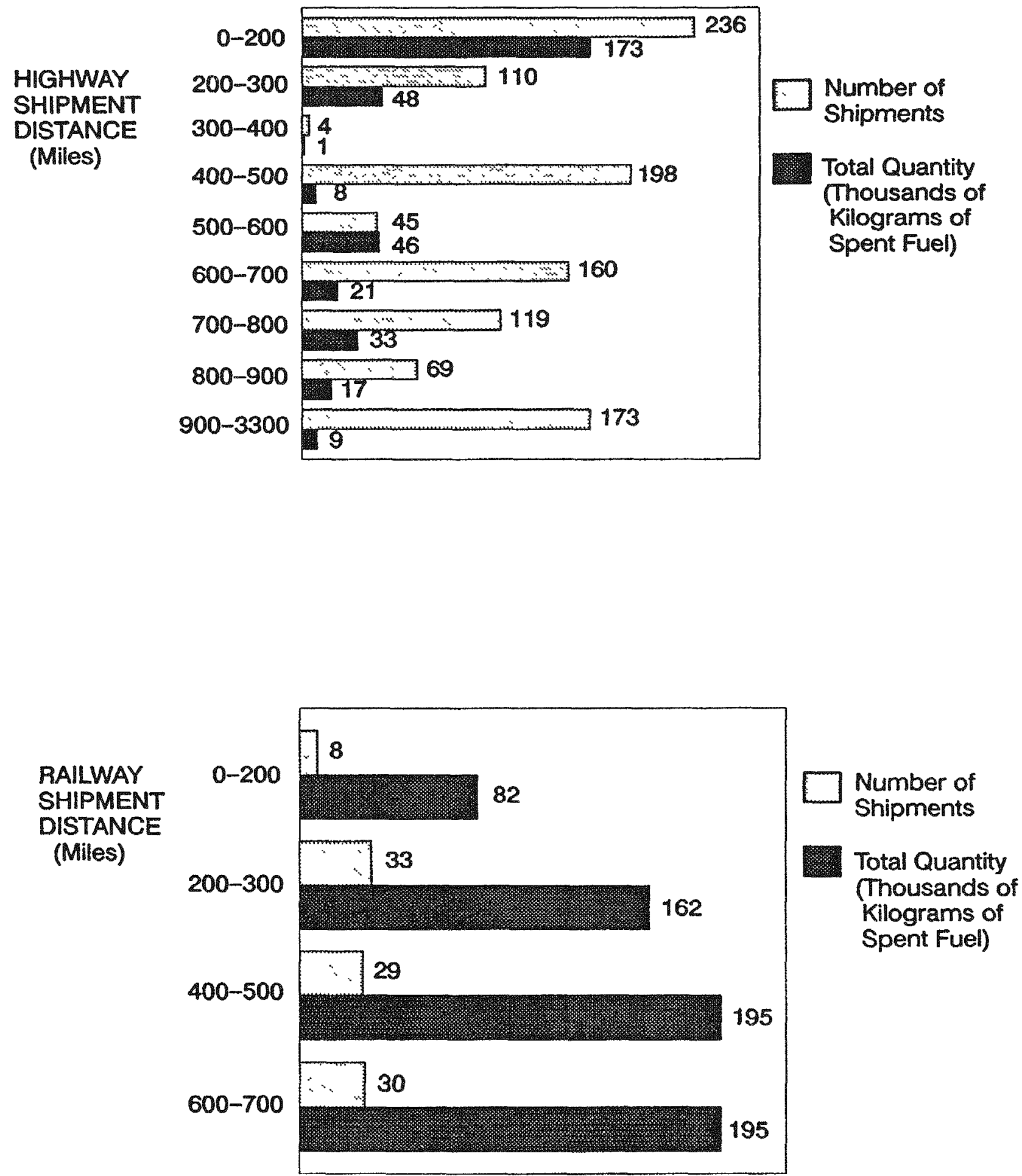

Figure 3.11 Number and Total Quantity of Spent Fuel Shipments by Shipment Distance Range: 1979-1991 
Table 4.1 Highway and Railway Spent Fuel Shipment Routes Used in 1987-1991*

\begin{tabular}{|c|c|c|c|c|c|}
\hline \multirow[b]{2}{*}{ State } & \multirow[b]{2}{*}{ Shipment } & \multicolumn{3}{|c|}{ Highway Shipment Route Segment } & \multirow{2}{*}{$\begin{array}{l}\text { Shipments } \\
\text { Completed }\end{array}$} \\
\hline & & From: & Route & To: & \\
\hline $\mathrm{AZ}$ & $\begin{array}{l}\text { General Atomics to } \\
\text { Idaho National } \\
\text { Engineering Laboratory } \\
\text { (INEL) }\end{array}$ & NV line & $\mathrm{I}-15 \mathrm{~N}$ & UT line & 1987 \\
\hline $\mathrm{AR}$ & $\begin{array}{l}\text { Arkansas One to } \\
\text { Portsmouth, Virginia } \\
\text { Marine Terminal }\end{array}$ & $\begin{array}{l}\text { Plant } \\
\text { AR-333 } \\
\text { US-64 } \\
\text { AR-326 }\end{array}$ & $\begin{array}{l}\text { AR-333 N } \\
\text { US-64 E } \\
\text { AR-326 N } \\
\text { I-40 E }\end{array}$ & $\begin{array}{l}\text { US-64 } \\
\text { AR-326 } \\
\text { I-40 } \\
\text { TN Line }\end{array}$ & 1991 \\
\hline \multirow[t]{5}{*}{$\mathrm{CA}$} & $\begin{array}{l}\text { University of Calif. } \\
\text { Berkeley (UCB) to Idaho } \\
\text { National Engineering } \\
\text { Laboratory }\end{array}$ & $\begin{array}{l}\text { UCB } \\
\text { private road } \\
\text { I-80 } \\
\text { I-880 } \\
\text { I-238 } \\
\text { I-580 } \\
\text { I-205 } \\
\text { I-5 }\end{array}$ & $\begin{array}{l}\text { private road W } \\
\mathrm{I}-80 \mathrm{~S} \\
\mathrm{I}-880 \mathrm{~S} \\
\mathrm{I}-238 \mathrm{E} \\
\mathrm{I}-580 \mathrm{E} \\
\mathrm{I}-205 \mathrm{E} \\
\mathrm{I}-5 \mathrm{~N} \\
\mathrm{I}-80 \mathrm{E}\end{array}$ & $\begin{array}{l}\text { I-80 } \\
\text { I-880 } \\
\text { I-238 } \\
\text { I-580 } \\
\text { I-205 } \\
\text { I-5 } \\
\text { I-80 } \\
\text { NV Line }\end{array}$ & 1989 \\
\hline & $\begin{array}{l}\text { Portsmouth, Virginia, } \\
\text { Marine Terminal to } \\
\text { GE/Vallecitos }\end{array}$ & $\begin{array}{l}\text { NV line } \\
\text { I-80 } \\
\text { I-5 } \\
\text { I-205 } \\
\text { I-580 } \\
\text { I-680 }\end{array}$ & $\begin{array}{l}\mathrm{I}-80 \mathrm{~W} \\
\mathrm{I}-5 \mathrm{~S} \\
\mathrm{I}-205 \mathrm{~W} \\
\mathrm{I}-580 \mathrm{~W} \\
\mathrm{I}-680 \mathrm{E} \\
\mathrm{CA}-84 \mathrm{E}\end{array}$ & $\begin{array}{l}\mathrm{I}-5 \\
\mathrm{I}-205 \\
\mathrm{I}-580 \\
\mathrm{I}-680 \\
\text { CA-84 } \\
\text { GE/VAL }\end{array}$ & 1988 \\
\hline & $\begin{array}{l}\text { GE/Vallecitos to } \\
\text { Portland Marine } \\
\text { Terminal }\end{array}$ & $\begin{array}{l}\text { GE/VAL } \\
\text { CA-84 } \\
\text { I-680 } \\
\text { I-580 } \\
\text { I-205 }\end{array}$ & $\begin{array}{l}\mathrm{CA}-84 \mathrm{~W} \\
\mathrm{I}-680 \mathrm{~N} \\
\mathrm{I}-580 \mathrm{E} \\
\mathrm{I}-205 \mathrm{E} \\
\mathrm{I}-5 \mathrm{~N}\end{array}$ & $\begin{array}{l}\text { I-680 } \\
\text { I-580 } \\
\text { I-205 } \\
\text { I-5 } \\
\text { OR line }\end{array}$ & 1988 \\
\hline & $\begin{array}{l}\text { GE/Vallecitos to } \\
\text { Port of Oakland }\end{array}$ & $\begin{array}{l}\text { GE/VAL } \\
\text { CA-84 } \\
\text { I-680 } \\
\text { I-580 } \\
\text { CA-238 } \\
\text { I-880 } \\
\text { Market St. } \\
\text { 3rd St. } \\
\text { Middle } \\
\text { Harbor Rd. }\end{array}$ & $\begin{array}{l}\text { CA-84 W } \\
\text { I-680 N } \\
\text { I-580 W } \\
\text { CA-238 W } \\
\text { I-880 N } \\
\text { Market St. W } \\
\text { 3rd St. N. } \\
\text { Middle Harbor } \\
\text { Rd. W } \\
\text { 7th St. W }\end{array}$ & $\begin{array}{l}\text { I-680 } \\
\text { I-580 } \\
\text { CA-238 } \\
\text { I-880 } \\
\text { Market St. } \\
\text { 3rd St. } \\
\text { Middle } \\
\text { Harbor Rd. } \\
\text { 7th St. } \\
\text { 5190 7th St. } \\
\text { Term.** } \\
\text { Berth }\end{array}$ & 1987 \\
\hline & $\begin{array}{l}\text { Port of Oakland to } \\
\text { GE/Vallecitos }\end{array}$ & $\begin{array}{l}\text { Oakland Term. } \\
\text { Market St. } \\
\text { 5th St. } \\
\text { I-880 } \\
\text { CA-238 } \\
\text { I-580 } \\
\text { I-680 }\end{array}$ & $\begin{array}{l}\text { Market St. N } \\
\text { 5th St. S } \\
\text { I-880 S } \\
\text { CA-238 E } \\
\text { I-580 E } \\
\text { I-680 S } \\
\text { CA-84 E }\end{array}$ & $\begin{array}{l}\text { 5th St. } \\
\text { I-880 } \\
\text { CA-238 } \\
1-580 \\
\text { I-680 } \\
\text { CA-84 } \\
\text { GE/VAL }\end{array}$ & 1988 \\
\hline
\end{tabular}


Table 4.1 (Continued)

\begin{tabular}{|c|c|c|c|c|c|}
\hline \multirow[b]{2}{*}{ State } & \multirow[b]{2}{*}{ Shipment } & \multicolumn{3}{|c|}{ Highway Shipment Route Segment } & \multirow{2}{*}{$\begin{array}{l}\text { Shipments } \\
\text { Completed }\end{array}$} \\
\hline & & From: & Route & To: & \\
\hline \multirow[t]{2}{*}{$\begin{array}{l}\text { CA } \\
\text { (Cont.) } \\
\end{array}$} & $\begin{array}{l}\text { General Atomics to } \\
\text { Idaho National } \\
\text { Engineering } \\
\text { Laboratory }\end{array}$ & $\begin{array}{l}\text { GA } \\
\text { local road } \\
\text { I-5 } \\
\text { CA-55 } \\
\text { CA-91 } \\
\text { I-215 }\end{array}$ & $\begin{array}{l}\text { local road E } \\
I-5 \mathrm{~N} \\
\mathrm{CA}-55 \mathrm{~N} \\
\mathrm{CA}-91 \mathrm{E} \\
\mathrm{I}-215 \mathrm{~N} \\
\mathrm{I}-15 \mathrm{~N}\end{array}$ & $\begin{array}{l}\text { I-5 } \\
\text { CA-55 } \\
\text { CA-91 } \\
\text { I-215 } \\
\text { I-15 } \\
\text { NV line }\end{array}$ & 1987 \\
\hline & $\begin{array}{l}\text { Babcock \& Wilcox, } \\
\text { Lynchburg, to } \\
\text { GE/Vallecitos }\end{array}$ & $\begin{array}{l}\text { NV line } \\
\mathrm{I}-80 \\
\mathrm{I}-5 \\
\mathrm{I}-205 \\
\mathrm{I}-580 \\
\mathrm{I}-680\end{array}$ & $\begin{array}{l}I-80 \mathrm{~W} \\
\mathrm{I}-5 \mathrm{~S} \\
\mathrm{I}-205 \mathrm{~W} \\
\mathrm{I}-580 \mathrm{~W} \\
\mathrm{I}-680 \mathrm{~S} \\
\mathrm{CA}-84 \mathrm{E}\end{array}$ & $\begin{array}{l}\text { I-5 } \\
\text { I-205 } \\
\text { I-580 } \\
\text { I-680 } \\
\text { CA-84 } \\
\text { GE/VAL }\end{array}$ & 1987 \\
\hline \multirow[t]{2}{*}{$\mathrm{CO}$} & $\begin{array}{l}\text { Michigan State Univer- } \\
\text { sity (MSU) to Denver } \\
\text { Federal Center }\end{array}$ & $\begin{array}{l}\text { WY line } \\
\text { I-25 }\end{array}$ & $\begin{array}{l}\text { I-25 S } \\
\text { 6th Ave. W. }\end{array}$ & $\begin{array}{l}\text { Exit } 209 \\
\text { Denver } \\
\text { 6th Ave. } \\
\text { Denver } \\
\text { Fed. Ctr. }\end{array}$ & 1989 \\
\hline & $\begin{array}{l}\text { Fort St. Vrain Reactor } \\
\text { to Idaho National Engineering } \\
\text { Laboratory }\end{array}$ & $\begin{array}{l}\text { Fort St. Vrain } \\
\text { WCR } 19.5 \\
\text { WCR } 34 \\
\text { WCR } 19 \\
\text { CO-66 }\end{array}$ & $\begin{array}{l}\text { Weld Co. Rd. } \\
19.5 \text { S } \\
\text { WCR } 34 \text { W } \\
\text { WCR } 19 \\
\text { CO-66 W } \\
\text { I-25 N }\end{array}$ & $\begin{array}{l}\text { WCR } 34 \\
\text { WCR } 19 \\
\text { CO-66 } \\
\text { I-25 } \\
\text { WY line }\end{array}$ & 1991 \\
\hline GA & $\begin{array}{l}\text { Port of Savannah to } \\
\text { Savannah River Project } \\
\text { (SRP) }\end{array}$ & $\begin{array}{l}\text { Terminal } \\
\text { Port Roads } \\
\text { GA-17 } \\
\text { GA-21 } \\
\text { I-16 }\end{array}$ & $\begin{array}{l}\text { Port Roads W } \\
\text { GA-17 S } \\
\text { GA-21 S } \\
\text { I-16 W } \\
\text { I-95 N }\end{array}$ & $\begin{array}{l}\text { GA-17 } \\
\text { GA-21 } \\
\text { I-16 } \\
\text { I-95 } \\
\text { SC line }\end{array}$ & $1987 / 88$ \\
\hline \multirow[t]{5}{*}{ ID } & $\begin{array}{l}\text { University of Missouri } \\
\text { to Idaho National } \\
\text { Engineering Laboratory }\end{array}$ & $\begin{array}{l}\text { UT line } \\
\text { I-15 } \\
\text { US-26 }\end{array}$ & $\begin{array}{l}\mathrm{I}-15 \mathrm{~N} \\
\mathrm{US}-26 \mathrm{~N} \\
\mathrm{US}-20 \mathrm{~W}\end{array}$ & $\begin{array}{l}\text { US-26 } \\
\text { US-20 } \\
\text { INEL }\end{array}$ & 1989 \\
\hline & $\begin{array}{l}\text { Michigan State } \\
\text { University to Idaho } \\
\text { National Engineering } \\
\text { Laboratory }\end{array}$ & & \multicolumn{2}{|c|}{ (same as above route) } & 1989 \\
\hline & $\begin{array}{l}\text { University of Calif. } \\
\text { Berkeley to Idaho } \\
\text { National Engineering } \\
\text { Laboratory }\end{array}$ & & \multicolumn{2}{|c|}{ (same as above route) } & 1989 \\
\hline & $\begin{array}{l}\text { General Atomics to } \\
\text { Idaho National } \\
\text { Engineering Laboratory }\end{array}$ & & \multicolumn{2}{|c|}{ (same as above route) } & 1987 \\
\hline & $\begin{array}{l}\text { Fort St. Vrain to Idaho } \\
\text { National Engineering } \\
\text { Laboratory }\end{array}$ & & \multicolumn{2}{|c|}{ (same as above route) } & 1991 \\
\hline
\end{tabular}


Table 4.1 (Continued)

\begin{tabular}{|c|c|c|c|c|c|}
\hline \multirow[b]{2}{*}{ State } & \multirow[b]{2}{*}{ Shipment } & \multicolumn{3}{|c|}{ Highway Shipment Route Segment } & \multirow{2}{*}{$\begin{array}{l}\text { Shipments } \\
\text { Completed }\end{array}$} \\
\hline & & From: & Route & To: & \\
\hline $\begin{array}{l}\text { ID } \\
\text { (Cont.) }\end{array}$ & $\begin{array}{l}\text { Idaho National Engineering } \\
\text { Laboratory to Portland } \\
\text { Marine Terminal }\end{array}$ & $\begin{array}{l}\text { Plant } \\
\text { US-26 } \\
\text { I-15 } \\
\text { I-86 }\end{array}$ & $\begin{array}{l}\text { US-26 E } \\
\mathrm{I}-15 \mathrm{~S} \\
\mathrm{I}-86 \mathrm{~W} / \mathrm{S} \\
\mathrm{I}-84 \mathrm{~W}\end{array}$ & $\begin{array}{l}\text { I-15 } \\
\text { I-86 } \\
\text { I-84 } \\
\text { OR Line }\end{array}$ & 1991 \\
\hline \multirow[t]{11}{*}{ IL } & $\begin{array}{l}\text { Michigan State } \\
\text { University to Idaho } \\
\text { National Engineering } \\
\text { Laboratory }\end{array}$ & $\begin{array}{l}\text { IN line } \\
\mathrm{I}-80\end{array}$ & $\begin{array}{l}\mathrm{I}-80 \mathrm{~W} \\
\mathrm{I}-280 \mathrm{~W}\end{array}$ & $\begin{array}{l}\text { I-280 } \\
\text { IA line }\end{array}$ & 1989 \\
\hline & $\begin{array}{l}\text { Michigan State } \\
\text { University to Denver } \\
\text { Federal Center }\end{array}$ & & (same as above route) & & 1989 \\
\hline & $\begin{array}{l}\text { Callaway to } \\
\text { Alexandria Bay }\end{array}$ & $\begin{array}{l}\text { MO line } \\
\mathrm{I}-270\end{array}$ & $\begin{array}{l}\mathrm{I}-270 \mathrm{E} \\
\mathrm{I}-70 \mathrm{E}\end{array}$ & $\begin{array}{l}\text { I-70 } \\
\text { IN line }\end{array}$ & 1991 \\
\hline & $\begin{array}{l}\text { University of Missouri } \\
\text { to Savannah River } \\
\text { Project }\end{array}$ & $\begin{array}{l}\text { MO line } \\
\text { I-255 } \\
\text { I-64 } \\
\text { I-57 }\end{array}$ & $\begin{array}{l}\mathrm{I}-255 \mathrm{E} \\
\mathrm{I}-64 \mathrm{E} \\
\mathrm{I}-57 \mathrm{~S} \\
\mathrm{I}-24 \mathrm{~S}\end{array}$ & $\begin{array}{l}\text { I- }-64 \\
\text { I-57 } \\
\text { I-24 } \\
\text { KY line }\end{array}$ & 1987 \\
\hline & $\begin{array}{l}\text { Dresden Reactor to } \\
\text { Portsmouth, Virginia, } \\
\text { Marine Terminal }\end{array}$ & $\begin{array}{l}\text { Dresden } \\
\text { Lorenzo Rd. } \\
\text { I-55 }\end{array}$ & $\begin{array}{l}\text { Lorenzo Rd. E } \\
\mathrm{I}-55 \mathrm{~S} \\
\mathrm{I}-74 \mathrm{E}\end{array}$ & $\begin{array}{l}\text { I-55 } \\
\text { I-74 } \\
\text { IN line }\end{array}$ & 1987 \\
\hline & $\begin{array}{l}\text { Dresden Reactor to } \\
\text { Babcock \& Wilcox, } \\
\text { Lynchburg }\end{array}$ & & (same as above route) & & 1987 \\
\hline & $\begin{array}{l}\text { Babcock \& Wilcox, } \\
\text { Lynchburg, to } \\
\text { GE/Vallecitos }\end{array}$ & $\begin{array}{l}\text { IN line } \\
\text { I-74 } \\
\text { I-274 } \\
\text { I-74 }\end{array}$ & $\begin{array}{l}\mathrm{I}-74 \mathrm{~W} \\
\mathrm{I}-274 \mathrm{~W} \\
\mathrm{I}-74 \mathrm{~W} \\
\mathrm{I}-280 \mathrm{~W}\end{array}$ & $\begin{array}{l}\text { I-274 } \\
\text { I-74 } \\
\text { I-280 } \\
\text { IA line }\end{array}$ & 1987 \\
\hline & $\begin{array}{l}\text { Portsmouth, VA, Marine } \\
\text { Terminal to GE/Vallecitos }\end{array}$ & & (same as above route) & & 1988 \\
\hline & $\begin{array}{l}\text { Babcock \& Wilcox, } \\
\text { Lynchburg, to Quad } \\
\text { Cities }\end{array}$ & $\begin{array}{l}\text { IN line } \\
\text { I- } 74 \\
\text { I- } 474 \\
\text { I- } 74 \\
\text { IL }-80\end{array}$ & $\begin{array}{l}\mathrm{I}-74 \mathrm{~W} \\
\mathrm{I}-474 \mathrm{~W} \\
\mathrm{I}-74 \mathrm{~W} \\
\mathrm{I}-80 \mathrm{~N} \\
\mathrm{IL}-84 \mathrm{~N}\end{array}$ & $\begin{array}{l}\text { I-474 } \\
\text { I-74 } \\
\text { I-80 } \\
\text { IL-84 } \\
\text { Quad Cities }\end{array}$ & 1987 \\
\hline & $\begin{array}{l}\text { Battelle Columbus to } \\
\text { GE/Morris }\end{array}$ & $\begin{array}{l}\text { IN line } \\
\text { I-74 } \\
\text { I-55 } \\
\text { Lorenzo Rd. }\end{array}$ & $\begin{array}{l}\text { I-74 W } \\
\text { I-55 N } \\
\text { Lorenzo Rd. W } \\
\text { Dresden Rd. N }\end{array}$ & $\begin{array}{l}\text { I-55 } \\
\text { Lorenzo Rd. } \\
\text { Dresden Rd. } \\
\text { GE/Morris }\end{array}$ & 1987 \\
\hline & $\begin{array}{l}\text { Byron Station } \\
\text { to Alexandria Bay }\end{array}$ & $\begin{array}{l}\text { Byron } \\
\text { German Church } \\
\text { Road } \\
\text { IL-64 } \\
\text { I-39 }\end{array}$ & $\begin{array}{l}\text { German Church Rd. } \\
\text { IL-64 } \\
\text { I-39 S } \\
\text { I-80 E }\end{array}$ & $\begin{array}{l}\text { IL-64 } \\
\text { I-39 } \\
\text { I-80 } \\
\text { IN line }\end{array}$ & 1991 \\
\hline
\end{tabular}


Table 4.1 (Continued)

\begin{tabular}{|c|c|c|c|c|c|}
\hline \multirow[b]{2}{*}{ State } & \multirow[b]{2}{*}{ Shipment } & \multicolumn{3}{|c|}{ Highway Shipment Route Segment } & \multirow{2}{*}{$\begin{array}{l}\text { Shipments } \\
\text { Completed }\end{array}$} \\
\hline & & From: & Route & To: & \\
\hline \multirow[t]{10}{*}{ IN } & $\begin{array}{l}\text { Michigan State } \\
\text { University to Idaho } \\
\text { National Engineering } \\
\text { Laboratory }\end{array}$ & $\begin{array}{l}\text { MI line } \\
I-69\end{array}$ & $\begin{array}{l}\mathrm{I}-69 \mathrm{~S} \\
\mathrm{I}-80 \mathrm{~W}\end{array}$ & $\begin{array}{l}\text { I-80 } \\
\text { IL line }\end{array}$ & 1989 \\
\hline & $\begin{array}{l}\text { Michigan State } \\
\text { University to Denver } \\
\text { Federal Center }\end{array}$ & & (same as abov & & 1989 \\
\hline & $\begin{array}{l}\text { Portsmouth, Virginia, } \\
\text { Marine Terminal to } \\
\text { GE/Vallecitos }\end{array}$ & $\begin{array}{l}\text { OH line } \\
\text { I-70 } \\
\text { I-465 }\end{array}$ & $\begin{array}{l}\mathrm{I}-70 \mathrm{~W} \\
\mathrm{I}-465 \mathrm{~N} \\
\mathrm{I}-74 \mathrm{~W}\end{array}$ & $\begin{array}{l}\text { I-465 } \\
\text { I-74 } \\
\text { IL line }\end{array}$ & 1988 \\
\hline & $\begin{array}{l}\text { Dresden Reactor to } \\
\text { Portsmouth, Virginia, } \\
\text { Marine Terminal }\end{array}$ & $\begin{array}{l}\text { IL line } \\
\text { I-74 } \\
\text { I-465 }\end{array}$ & $\begin{array}{l}I-74 \mathrm{E} \\
\mathrm{I}-465 \mathrm{~S} \\
\mathrm{I}-70 \mathrm{E}\end{array}$ & $\begin{array}{l}\text { I }-465 \\
\text { I-70 } \\
\text { OH line }\end{array}$ & 1987 \\
\hline & $\begin{array}{l}\text { Dresden Reactor to } \\
\text { Babcock \& Wilcox, } \\
\text { Lynchburg }\end{array}$ & & (same as abov & & 1987 \\
\hline & $\begin{array}{l}\text { Babcock \& Wilcox, } \\
\text { Lynchburg, to } \\
\text { GE/Vallecitos }\end{array}$ & $\begin{array}{l}\text { OH line } \\
\text { I-70 } \\
\text { I-465 }\end{array}$ & $\begin{array}{l}\mathrm{I}-70 \mathrm{~W} \\
\mathrm{I}-465 \mathrm{~S} \\
\mathrm{I}-74 \mathrm{~W}\end{array}$ & $\begin{array}{l}I-465 \\
\text { I-74 } \\
\text { IL line }\end{array}$ & 1987 \\
\hline & $\begin{array}{l}\text { Battelle Columbus to } \\
\text { GE/Morris }\end{array}$ & & (same as abov & & 1987 \\
\hline & $\begin{array}{l}\text { Babcock \& Wilcox, } \\
\text { Lynchburg, to Quad } \\
\text { Cities }\end{array}$ & $\begin{array}{l}\text { KY line } \\
\text { I-65 } \\
\text { I-465 }\end{array}$ & $\begin{array}{l}\mathrm{I}-65 \mathrm{~N} \\
\mathrm{I}-465 \mathrm{~W} \\
\mathrm{I}-74 \mathrm{~W}\end{array}$ & $\begin{array}{l}\text { I-465 } \\
\text { I-74 } \\
\text { IL line }\end{array}$ & 1987 \\
\hline & Byron to Alexandria Bay & IL line & $\mathrm{I}-80 \mathrm{E}$ & OH line & 1991 \\
\hline & $\begin{array}{l}\text { Callaway to Alexandria } \\
\text { Bay }\end{array}$ & $\begin{array}{l}\text { IL line } \\
\text { I-70 } \\
\text { I-465 }\end{array}$ & $\begin{array}{l}\mathrm{I}-70 \mathrm{~N} / \mathrm{E} \\
\mathrm{I}-465 \mathrm{~S} / \mathrm{E} / \mathrm{N} \\
\mathrm{I}-70 \mathrm{E}\end{array}$ & $\begin{array}{l}\text { I }-465 \\
\text { I-70 } \\
\text { OH line }\end{array}$ & 1991 \\
\hline \multirow[t]{4}{*}{ IA } & $\begin{array}{l}\text { Michigan State } \\
\text { University to Idaho } \\
\text { National Engineering } \\
\text { Laboratory }\end{array}$ & $\begin{array}{l}\text { IL line } \\
\text { I-280 } \\
\text { I-80 } \\
\text { I-680 } \\
\text { I-29 }\end{array}$ & $\begin{array}{l}\mathrm{I}-280 \mathrm{~W} \\
\mathrm{I}-80 \mathrm{~W} \\
\mathrm{I}-680 \mathrm{~W} \\
\mathrm{I}-29 \mathrm{~S} \\
\mathrm{I}-680 \mathrm{~W}\end{array}$ & $\begin{array}{l}\text { I-80 } \\
\text { I-680 } \\
\text { I-29 } \\
\text { I-680 } \\
\text { NE line }\end{array}$ & 1989 \\
\hline & $\begin{array}{l}\text { Michigan State } \\
\text { University to Denver } \\
\text { Federal Center }\end{array}$ & & \multicolumn{2}{|c|}{ (same as above route) } & 1989 \\
\hline & $\begin{array}{l}\text { Babcock \& Wilcox, } \\
\text { Lynchburg, to } \\
\text { GE/Vallecitos }\end{array}$ & & \multicolumn{2}{|c|}{ (same as above route) } & 1987 \\
\hline & $\begin{array}{l}\text { Portsmouth, Virginia, } \\
\text { Marine Terminal to } \\
\text { GE/Vallecitos }\end{array}$ & & \multicolumn{2}{|c|}{ (same as above route) } & 1988 \\
\hline
\end{tabular}


Table 4.1 (Continued)

\begin{tabular}{|c|c|c|c|c|c|}
\hline \multirow[b]{2}{*}{ State } & \multirow[b]{2}{*}{ Shipment } & \multicolumn{3}{|c|}{ Highway Shipment Route Segment } & \multirow{2}{*}{$\begin{array}{l}\text { Shipments } \\
\text { Completed }\end{array}$} \\
\hline & & From: & Route & To: & \\
\hline \multirow[t]{3}{*}{ KY } & $\begin{array}{l}\text { University of Michigan } \\
\text { to Savannah River } \\
\text { Project }\end{array}$ & $\begin{array}{l}\text { OH line } \\
\mathrm{I}-275\end{array}$ & $\begin{array}{l}\mathrm{I}-275 \mathrm{~S} \\
\mathrm{I}-75 \mathrm{~S}\end{array}$ & $\begin{array}{l}\text { I-75 } \\
\text { TN line }\end{array}$ & 1987 \\
\hline & $\begin{array}{l}\text { University of Missouri } \\
\text { to Savannah River } \\
\text { Project }\end{array}$ & IL line & $\mathrm{I}-24 \mathrm{~S}$ & TN line & 1987 \\
\hline & $\begin{array}{l}\text { Babcock \& Wilcox, } \\
\text { Lynchburg, to Quad } \\
\text { Cities }\end{array}$ & $\begin{array}{l}\text { TN line } \\
\mathrm{I}-75 \\
\mathrm{I}-64\end{array}$ & $\begin{array}{l}\mathrm{I}-75 \mathrm{~N} \\
\mathrm{I}-64 \mathrm{~W} \\
\mathrm{I}-65 \mathrm{~N}\end{array}$ & $\begin{array}{l}\text { I-64 } \\
\text { I-65 } \\
\text { IN line }\end{array}$ & 1987 \\
\hline \multirow[t]{9}{*}{$\mathrm{MD}$} & $\begin{array}{l}\text { Alexandria Bay to } \\
\text { Savannah River Project }\end{array}$ & PA line & I $-81 \mathrm{~S}$ & WV line & 1988 \\
\hline & $\begin{array}{l}\text { CINTICHEM to } \\
\text { Savannah River Project }\end{array}$ & & (same as above rc & & $1987 / 91$ \\
\hline & $\begin{array}{l}\text { Summer to Alexander } \\
\text { Bay }\end{array}$ & & (reverse of above & & 1990 \\
\hline & $\begin{array}{l}\text { Dresden Reactor to } \\
\text { Portsmouth, Virginia, } \\
\text { Marine Terminal }\end{array}$ & $\begin{array}{l}\text { WV line } \\
\text { US-48/US-40 } \\
\text { I-70 }\end{array}$ & $\begin{array}{l}\text { US-48/US-40 E } \\
\text { I-70 E } \\
\text { I-81 S }\end{array}$ & $\begin{array}{l}\text { I-70 } \\
\text { I-81 } \\
\text { WV line }\end{array}$ & 1987 \\
\hline & $\begin{array}{l}\text { Dresden Reactor to } \\
\text { Babcock \& Wilcox } \\
\text { Lynchburg }\end{array}$ & & (same as above $\mathrm{rc}$ & & 1987 \\
\hline & $\begin{array}{l}\text { Portsmouth, Virginia, } \\
\text { Marine Terminal to } \\
\text { GE/Vallecitos }\end{array}$ & & (reverse of above & & 1988 \\
\hline & $\begin{array}{l}\text { National Institute of } \\
\text { Standards and } \\
\text { Technology (NIST) to } \\
\text { Savannah River Project }\end{array}$ & $\begin{array}{l}\text { NIST } \\
\text { local roads } \\
\mathrm{I}-270 \\
\mathrm{I}-70\end{array}$ & $\begin{array}{l}\text { local roads E } \\
\text { I-270 N } \\
\mathrm{I}-70 \mathrm{~W} \\
\mathrm{I}-81 \mathrm{~S}\end{array}$ & $\begin{array}{l}\text { I-270 } \\
\text { I-70 } \\
\text { US-40/48 } \\
\text { WV line }\end{array}$ & 1987 \\
\hline & $\begin{array}{l}\text { Babcock \& Wilcox, } \\
\text { Lynchburg, to } \\
\text { GE/Vallecitos }\end{array}$ & $\begin{array}{l}\text { WV line } \\
\text { I-81 } \\
\text { I-70 }\end{array}$ & $\begin{array}{l}\text { I- } 81 \mathrm{~N} \\
\text { I-70 W } \\
\text { US-40/48 W }\end{array}$ & $\begin{array}{l}\text { I-70 } \\
\text { US-40/48 } \\
\text { WV line }\end{array}$ & 1987 \\
\hline & $\begin{array}{l}\text { Calvert Cliffs to } \\
\text { Alexandria Bay }\end{array}$ & $\begin{array}{l}\text { Calvert Cliffs } \\
\text { MD-4 } \\
\text { I-95 } \\
\mathrm{I}-495 \\
\mathrm{I}-270 \\
\mathrm{I}-70\end{array}$ & $\begin{array}{l}\mathrm{MD} 4 \mathrm{~N} \\
\mathrm{I}-95 \mathrm{~W} \\
\mathrm{I}-495 \mathrm{~W} \\
\mathrm{I}-270 \mathrm{~N} / \mathrm{W} \\
\mathrm{I}-70 \mathrm{~W} \\
\mathrm{I}-81 \mathrm{~N}\end{array}$ & $\begin{array}{l}\text { I-95 } \\
\text { I-495 } \\
\text { I-270 } \\
\text { I-70 } \\
\text { I-81 } \\
\text { PA line }\end{array}$ & 1990 \\
\hline \multirow[t]{2}{*}{ MI } & $\begin{array}{l}\text { Michigan State } \\
\text { University to Idaho } \\
\text { National Engineering } \\
\text { Laboratory }\end{array}$ & $\begin{array}{l}\text { MSU } \\
\text { local roads } \\
\text { US-27 } \\
\text { I-96 } \\
\text { Temp. I-69 }\end{array}$ & $\begin{array}{l}\text { local roads W } \\
\text { US }-127 \mathrm{~S} \\
\text { I-96 W } \\
\text { Temp. I-69 S } \\
\text { I-69 S }\end{array}$ & $\begin{array}{l}\text { US-127 } \\
\text { I-96 } \\
\text { Temp. I-69 } \\
\text { I-69 } \\
\text { IN line }\end{array}$ & 1989 \\
\hline & $\begin{array}{l}\text { Michigan State } \\
\text { University to Denver } \\
\text { Federal Center }\end{array}$ & & \multicolumn{2}{|c|}{ (same as above route) } & 1989 \\
\hline
\end{tabular}


Table 4.1 (Continued)

\begin{tabular}{|c|c|c|c|c|c|}
\hline \multirow[b]{2}{*}{ State } & \multirow[b]{2}{*}{ Shipment } & \multicolumn{3}{|c|}{ Highway Shipment Route Segment } & \multirow{2}{*}{$\begin{array}{l}\text { Shipments } \\
\text { Completed }\end{array}$} \\
\hline & & From: & Route & To: & \\
\hline $\begin{array}{l}\text { MI } \\
\text { (Cont.) }\end{array}$ & $\begin{array}{l}\text { University of Michigan } \\
\text { to Savannah River } \\
\text { Project }\end{array}$ & $\begin{array}{l}\text { University } \\
\text { of Michigan } \\
\text { Plymouth Rd } \\
\text { US-23 } \\
\text { I-94 } \\
\text { I-275 }\end{array}$ & $\begin{array}{l}\text { Plymouth Rd N } \\
\text { US-23 S } \\
\text { I-94 E } \\
\text { I-275 S } \\
\text { I-75 S }\end{array}$ & $\begin{array}{l}\text { US-23 } \\
\text { I-94 } \\
\text { I-275 } \\
\text { I-75 } \\
\text { OH line }\end{array}$ & 1987 \\
\hline \multirow[t]{3}{*}{ MO } & $\begin{array}{l}\text { University of Missouri } \\
\text { to Savannah River } \\
\text { Project }\end{array}$ & 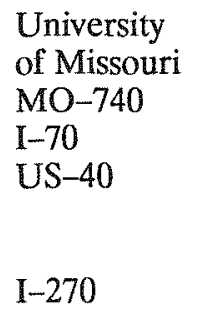 & $\begin{array}{l}\text { MO-740 W } \\
\text { I-70 E } \\
\text { US-40 S } \\
\text { I-270 S }\end{array}$ & $\begin{array}{l}\text { I-70 } \\
\text { US-40 } \\
\text { I-270 } \\
\text { I-255 } \\
\text { (contin. } \\
\text { of I-270) } \\
\text { IL line }\end{array}$ & 1987 \\
\hline & $\begin{array}{l}\text { University of Missouri } \\
\text { to Idaho National } \\
\text { Engineering Laboratory }\end{array}$ & $\begin{array}{l}\text { University } \\
\text { of Missouri } \\
\text { MO-740 } \\
\text { I-70 } \\
\text { I-435 }\end{array}$ & $\begin{array}{l}\mathrm{MO}-740 \mathrm{~W} \\
\mathrm{I}-70 \mathrm{~W} \\
\mathrm{I}-435 \mathrm{~N} \\
\mathrm{I}-29 \mathrm{~N}\end{array}$ & $\begin{array}{l}I-70 \\
I-435 \\
\text { I-29 } \\
\text { IA line }\end{array}$ & 1989 \\
\hline & $\begin{array}{l}\text { Callaway to } \\
\text { Alexandria Bay }\end{array}$ & $\begin{array}{l}\text { Callaway } \\
\mathrm{MO}-\mathrm{CC} \\
\mathrm{MO}-0 \\
\mathrm{MO}-\mathrm{D} \\
\mathrm{I}-70\end{array}$ & $\begin{array}{l}\text { MO-CC N } \\
\text { MO-0 E } \\
\text { MO-D N } \\
\text { I-70 E } \\
I-270 \mathrm{E}\end{array}$ & $\begin{array}{l}\mathrm{MO}-0 \\
\mathrm{MO}-\mathrm{D} \\
\mathrm{I}-70 \\
\mathrm{I}-270 \\
\text { IL line }\end{array}$ & 1991 \\
\hline \multirow[t]{5}{*}{$\mathrm{NE}$} & $\begin{array}{l}\text { Michigan State } \\
\text { University to Idaho } \\
\text { National Engineering } \\
\text { Laboratory }\end{array}$ & $\begin{array}{l}\text { IA line } \\
\text { I-680 }\end{array}$ & $\begin{array}{l}\text { I-680 W } \\
\text { I- } 80 \mathrm{~W}\end{array}$ & $\begin{array}{l}\text { I-80 } \\
\text { WY line }\end{array}$ & 1989 \\
\hline & $\begin{array}{l}\text { University of Missouri } \\
\text { to Idaho National } \\
\text { Engineering Laboratory }\end{array}$ & & \multicolumn{2}{|c|}{ (same as above route) } & 1989 \\
\hline & $\begin{array}{l}\text { Michigan State } \\
\text { University to Denver } \\
\text { Federal Center }\end{array}$ & & \multicolumn{2}{|c|}{ (same as above route) } & 1989 \\
\hline & $\begin{array}{l}\text { Babcock \& Wilcox, } \\
\text { Lynchburg, to } \\
\text { GE/Vallecitos }\end{array}$ & & \multicolumn{2}{|c|}{ (same as above route) } & 1987 \\
\hline & $\begin{array}{l}\text { Portsmouth, Virginia, } \\
\text { Marine Terminal to } \\
\text { GE/Vallecitos }\end{array}$ & & \multicolumn{2}{|c|}{ (same as above route) } & 1988 \\
\hline \multirow[t]{2}{*}{ NV } & $\begin{array}{l}\text { University of Calif. } \\
\text { Berkeley to Idaho } \\
\text { National Engineering } \\
\text { Laboratory }\end{array}$ & CA line & $\mathrm{I}-80 \mathrm{E}$ & UT line & 1989 \\
\hline & $\begin{array}{l}\text { Portsmouth, Virginia, } \\
\text { Marine Terminal to } \\
\text { GE/Vallecitos }\end{array}$ & & \multicolumn{2}{|c|}{ (reverse of above route) } & 1988 \\
\hline
\end{tabular}


Table 4.1 (Continued)

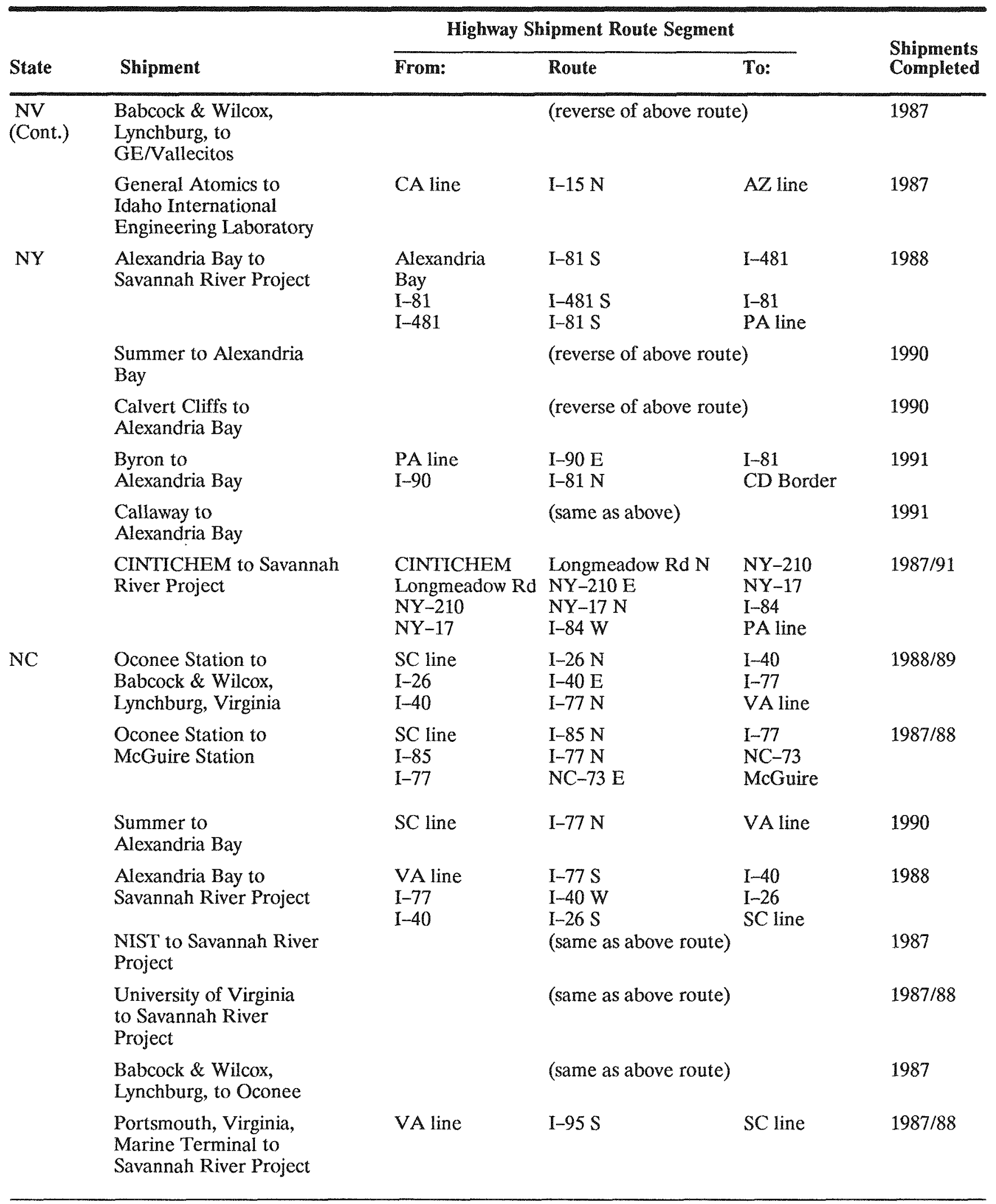


Table 4.1 (Continued)

\begin{tabular}{|c|c|c|c|c|c|}
\hline \multirow[b]{2}{*}{ State } & \multirow[b]{2}{*}{ Shipment } & \multicolumn{3}{|c|}{ Highway Shipment Route Segment } & \multirow{2}{*}{$\begin{array}{l}\text { Shipments } \\
\text { Completed }\end{array}$} \\
\hline & & From: & Route & To: & \\
\hline \multirow[t]{6}{*}{$\begin{array}{l}\mathrm{NC} \\
\text { (Cont.) }\end{array}$} & $\begin{array}{l}\text { Norfolk Int'l Term. to } \\
\text { Savannah River Project }\end{array}$ & & \multicolumn{2}{|c|}{ (same as above route) } & 1987 \\
\hline & $\begin{array}{l}\text { Newport News to } \\
\text { Savannah River Project }\end{array}$ & & \multicolumn{2}{|c|}{ (same as above route) } & 1989 \\
\hline & $\begin{array}{l}\text { University of Michigan } \\
\text { to Savannah River Project }\end{array}$ & $\begin{array}{l}\text { TN line } \\
\mathbb{I}-40\end{array}$ & $\begin{array}{l}\mathrm{I}-40 \mathrm{E} \\
\mathrm{I}-26 \mathrm{E}\end{array}$ & $\begin{array}{l}\mathrm{I}-26 \\
\text { SC line }\end{array}$ & 1987 \\
\hline & $\begin{array}{l}\text { CINTICHEM to Savannah } \\
\text { River Project }\end{array}$ & & \multicolumn{2}{|c|}{ (same as above route) } & 1987 \\
\hline & $\begin{array}{l}\text { University of Missouri } \\
\text { to Savannah River Project }\end{array}$ & & \multicolumn{2}{|c|}{ (same as above route) } & 1987 \\
\hline & $\begin{array}{l}\text { CINTICHEM to } \\
\text { Savannah River Project }\end{array}$ & VA line & $\mathrm{I}-77 \mathrm{~S}$ & SC line & 1991 \\
\hline \multirow[t]{8}{*}{$\mathrm{OH}$} & $\begin{array}{l}\text { Portsmouth, Virginia, } \\
\text { Marine Terminal to } \\
\text { GE/Vallecitos }\end{array}$ & $\begin{array}{l}\text { WV line } \\
\mathrm{I}-470 \\
\mathrm{I}-70 \\
\mathrm{I}-270\end{array}$ & $\begin{array}{l}I-470 \mathrm{~W} \\
\mathrm{I}-70 \mathrm{~W} \\
\mathrm{I}-270 \mathrm{~N} \\
\mathrm{I}-70 \mathrm{~W}\end{array}$ & $\begin{array}{l}\text { I-70 } \\
\text { I- } 270 \\
\text { I-70 } \\
\text { IN line }\end{array}$ & 1988 \\
\hline & $\begin{array}{l}\text { Dresden Reactor to } \\
\text { Portsmouth, Virginia, } \\
\text { Marine Terminal }\end{array}$ & & \multicolumn{2}{|c|}{ (reverse of above route) } & 1987 \\
\hline & $\begin{array}{l}\text { Dresden Reactor to } \\
\text { Babcock \& Wilcox } \\
\text { Lynchburg }\end{array}$ & & \multicolumn{2}{|c|}{ (reverse of above route) } & 1987 \\
\hline & $\begin{array}{l}\text { University of Michigan } \\
\text { to Savannah River } \\
\text { Project }\end{array}$ & $\begin{array}{l}\text { MI line } \\
\text { I-75 } \\
I-475 \\
I-75\end{array}$ & $\begin{array}{l}I-75 S \\
I-475 S \\
I-75 S \\
I-275 E\end{array}$ & $\begin{array}{l}I-475 \\
I-75 \\
I-275 \\
K Y \text { line }\end{array}$ & 1987 \\
\hline & $\begin{array}{l}\text { Babcock \& Wilcox, } \\
\text { Lynchburg, to } \\
\text { GE/Vallecitos }\end{array}$ & $\begin{array}{l}\text { WV line } \\
I-470 \\
I-70 \\
I-270\end{array}$ & $\begin{array}{l}I-470 W \\
I-70 W \\
I-270 \mathrm{~S} \\
I-70 W\end{array}$ & $\begin{array}{l}I-70 \\
I-270 \\
I-70 \\
\text { IN line }\end{array}$ & 1987 \\
\hline & $\begin{array}{l}\text { Battelle Columbus to } \\
\text { GE/Morris }\end{array}$ & $\begin{array}{l}\text { Site } \\
\text { OH-142 } \\
\text { US-40 } \\
\text { US-29 }\end{array}$ & $\begin{array}{l}\text { OH-142 N } \\
\text { US-40 N } \\
\text { US-29 N } \\
\text { I-70 W }\end{array}$ & $\begin{array}{l}\text { US-40 } \\
\text { US-29 } \\
\text { I-7 } \\
\text { IN line }\end{array}$ & 1987 \\
\hline & $\begin{array}{l}\text { Byron to } \\
\text { Alexandria Bay }\end{array}$ & IL line & $\mathrm{I}-80 \mathrm{E}$ & PA line & 1991 \\
\hline & $\begin{array}{l}\text { Callaway to } \\
\text { Alexandria Bay }\end{array}$ & $\begin{array}{l}\text { IN line } \\
I-70 \\
I-270 \\
I-70\end{array}$ & $\begin{array}{l}I-70 \mathrm{E} \\
\mathrm{I}-270 \mathrm{~S} / \mathrm{E} / \mathrm{N} \\
\mathrm{I}-70 \mathrm{E} \\
\mathrm{I}-470 \mathrm{E}\end{array}$ & $\begin{array}{l}I-270 \\
I-70 \\
I-470 \\
\text { WV line }\end{array}$ & 1991 \\
\hline
\end{tabular}


Table 4.1 (Continued)

\begin{tabular}{|c|c|c|c|c|c|}
\hline \multirow[b]{2}{*}{ State } & \multirow[b]{2}{*}{ Shipment } & \multicolumn{3}{|c|}{ Highway Shipment Route Segment } & \multirow{2}{*}{$\begin{array}{l}\text { Shipments } \\
\text { Completed }\end{array}$} \\
\hline & & From: & Route & To: & \\
\hline \multirow[t]{2}{*}{$\overline{\mathrm{OR}}$} & $\begin{array}{l}\text { GE/Vallecitos to } \\
\text { Portland Marine } \\
\text { Terminal }\end{array}$ & $\begin{array}{l}\text { CA line } \\
\text { I-5 } \\
\text { I-205 } \\
\text { Airport Way } \\
\text { 122nd Ave. } \\
\text { Marine Dr. } \\
\text { Portland Rd. }\end{array}$ & $\begin{array}{l}\text { I-5 N } \\
\text { I-205 N } \\
\text { Airport Way E } \\
\text { 122nd Ave. N } \\
\text { Marine Dr. W } \\
\text { Portland Rd. S. } \\
\text { N. Marine Dr. W }\end{array}$ & $\begin{array}{l}\text { I-205 } \\
\text { (Exit 288) } \\
\text { Exit 24B } \\
\text { Airport Way } \\
\text { 122nd Ave. } \\
\text { Marine Dr. } \\
\text { Portland Rd. } \\
\text { N. Marine Dr. } \\
\text { Term. No. } 6\end{array}$ & 1989 \\
\hline & $\begin{array}{l}\text { Idaho National } \\
\text { Engineering Laboratory } \\
\text { to Portland } \\
\text { Marine Terminal }\end{array}$ & $\begin{array}{l}\text { IO Line } \\
\text { I-84 } \\
\text { Marine Dr. }\end{array}$ & $\begin{array}{l}\mathrm{I}-84 \mathrm{~W} / \mathrm{N} \\
\text { Marine Dr. W } \\
\text { Exit road }\end{array}$ & $\begin{array}{l}\text { Marine Dr. } \\
\text { Term. No. } 6 \text { Exit } \\
\text { Term. No. } 6\end{array}$ & 1991 \\
\hline \multirow[t]{10}{*}{ PA } & $\begin{array}{l}\text { Alexandria Bay to } \\
\text { Savannah River Project }\end{array}$ & NY line & $\mathrm{I}-81 \mathrm{~S}$ & MD line & 1988 \\
\hline & $\begin{array}{l}\text { Calvert Cliffs to } \\
\text { Alexandria Bay }\end{array}$ & & \multicolumn{2}{|c|}{ (reverse of above route) } & 1990 \\
\hline & $\begin{array}{l}\text { Summer to } \\
\text { Alexandria Bay }\end{array}$ & & \multicolumn{2}{|c|}{ (reverse of above route) } & 1990 \\
\hline & $\begin{array}{l}\text { Byron to } \\
\text { Alexandria Bay }\end{array}$ & $\begin{array}{l}\text { OH line } \\
\text { I-80 } \\
\text { I-79 }\end{array}$ & $\begin{array}{l}I-80 \mathrm{E} \\
\mathrm{I}-79 \mathrm{~N} \\
\mathrm{I}-90 \mathrm{~N} / \mathrm{E}\end{array}$ & $\begin{array}{l}\text { I-79 } \\
\text { I-90 } \\
\text { NY line }\end{array}$ & 1991 \\
\hline & $\begin{array}{l}\text { CINTICHEM to Savannah } \\
\text { River Project }\end{array}$ & $\begin{array}{l}\text { NY line } \\
\text { I-84 } \\
\mathrm{I}-380 \\
\mathrm{I}-80\end{array}$ & $\begin{array}{l}I-84 W \\
I-380 \mathrm{~S} \\
\mathrm{I}-80 \mathrm{~W} \\
\mathrm{I}-81 \mathrm{~S}\end{array}$ & $\begin{array}{l}\mathrm{I}-380 \\
\mathrm{I}-80 \\
\mathrm{I}-81 \\
\text { MD line }\end{array}$ & $1987 / 91$ \\
\hline & $\begin{array}{l}\text { Callaway to } \\
\text { Alexandria Bay }\end{array}$ & $\begin{array}{l}\text { WV line } \\
\text { I-70 } \\
\text { I-79 }\end{array}$ & $\begin{array}{l}\mathrm{I}-70 \mathrm{E} \\
\mathrm{I}-79 \mathrm{~N} \\
\mathrm{I}-90 \mathrm{E}\end{array}$ & $\begin{array}{l}\text { I-79 } \\
\text { I-90 } \\
\text { NY line }\end{array}$ & 1991 \\
\hline & $\begin{array}{l}\text { Dresden Reactor to } \\
\text { Portsmouth, Virginia, } \\
\text { Marine Terminal }\end{array}$ & $\begin{array}{l}\text { WV line } \\
\mathrm{I}-70\end{array}$ & $\begin{array}{l}\mathrm{I}-70 \mathrm{E} \\
\mathrm{I}-79 \mathrm{~S}\end{array}$ & $\begin{array}{l}\text { I-79 } \\
\text { WV line }\end{array}$ & 1987 \\
\hline & $\begin{array}{l}\text { Dresden Reactor to } \\
\text { Babcock \& Wilcox } \\
\text { Lynchburg }\end{array}$ & & \multicolumn{2}{|c|}{ (same as above route) } & 1987 \\
\hline & $\begin{array}{l}\text { Portsmouth, Virginia, } \\
\text { Marine Terminal to } \\
\text { GE/Vallecitos }\end{array}$ & & \multicolumn{2}{|c|}{ (reverse of above route) } & 1988 \\
\hline & $\begin{array}{l}\text { Babcock \& Wilcox, } \\
\text { Lynchburg to GE/VAL }\end{array}$ & & \multicolumn{2}{|c|}{ (reverse of above route) } & 1987 \\
\hline $\mathrm{SC}$ & $\begin{array}{l}\text { Oconee Station to } \\
\text { Babcock \& Wilcox, } \\
\text { Lynchburg }\end{array}$ & $\begin{array}{l}\text { Oconee } \\
\text { SC-130 } \\
\text { US-123 } \\
\text { US-76 } \\
\text { I-85 }\end{array}$ & $\begin{array}{l}\text { SC-130 S } \\
\text { US-123 E } \\
\text { US-76 S } \\
\text { I-85 N } \\
\mathrm{I}-26 \mathrm{~N}\end{array}$ & $\begin{array}{l}\text { US-123 } \\
\text { US-76 } \\
\text { I-85 } \\
\text { I-26 } \\
\text { NC line }\end{array}$ & $1988 / 89$ \\
\hline
\end{tabular}


Table 4.1 (Continued)

\begin{tabular}{|c|c|c|c|c|c|}
\hline \multirow[b]{2}{*}{ State } & \multirow[b]{2}{*}{ Shipment } & \multicolumn{3}{|c|}{ Highway Shipment Route Segment } & \multirow{2}{*}{$\begin{array}{l}\text { Shipments } \\
\text { Completed }\end{array}$} \\
\hline & & From: & Route & To: & \\
\hline \multirow[t]{13}{*}{$\begin{array}{l}\text { SC } \\
\text { (Cont.) }\end{array}$} & $\begin{array}{l}\text { Portsmouth, Virginia, } \\
\text { Marine Terminal to } \\
\text { Savannah River Project }\end{array}$ & $\begin{array}{l}\text { NC line } \\
\text { I-95 } \\
\text { US-301 } \\
\text { SC-70 }\end{array}$ & $\begin{array}{l}\text { I-95 S } \\
\text { US-301 W } \\
\text { SC-70 W } \\
\text { SC-64 W }\end{array}$ & $\begin{array}{l}\text { US-301 } \\
\text { SC-70 } \\
\text { SC-64 } \\
\text { SRP }\end{array}$ & $1987 / 88$ \\
\hline & $\begin{array}{l}\text { Norfolk Int'l Terminal } \\
\text { to Savannah River Project }\end{array}$ & & (same as abov & & 1987 \\
\hline & $\begin{array}{l}\text { Newport News to } \\
\text { Savannah River Project }\end{array}$ & & (same as abov & & 1989 \\
\hline & $\begin{array}{l}\text { Oconee Station to } \\
\text { McGuire Station }\end{array}$ & $\begin{array}{l}\text { Oconee } \\
\text { SC-130 } \\
\text { US-123 } \\
\text { US-76 }\end{array}$ & $\begin{array}{l}\text { SC-130S } \\
\text { US-123 E } \\
\text { US-76 S } \\
I-85 \mathrm{~N}\end{array}$ & $\begin{array}{l}\text { US-123 } \\
\text { US-76 } \\
\text { I-85 } \\
\text { NC line }\end{array}$ & $1987 / 88$ \\
\hline & $\begin{array}{l}\text { Alexandria Bay to } \\
\text { Savannah River Project }\end{array}$ & $\begin{array}{l}\text { NC Line } \\
\text { I-26 } \\
\text { SC-121 } \\
\text { SC-19 } \\
\text { SC-118 }\end{array}$ & $\begin{array}{l}\mathrm{I}-26 \mathrm{~S} \\
\mathrm{SC}-121 \mathrm{~S} \\
\mathrm{SC}-19 \mathrm{~S} \\
\mathrm{SC}-118 \mathrm{~W} \\
\mathrm{SC}-19 \mathrm{~S}\end{array}$ & $\begin{array}{l}\text { SC-121 } \\
\text { SC-19 } \\
\text { SC-118 } \\
\text { SC-19 } \\
\text { SRP }\end{array}$ & 1988 \\
\hline & $\begin{array}{l}\text { University of Michigan } \\
\text { to Savannah River } \\
\text { Project }\end{array}$ & & (same as abov & & 1987 \\
\hline & $\begin{array}{l}\text { CINTICHEM to Savannah } \\
\text { River Project }\end{array}$ & & (same as abov & & 1987 \\
\hline & $\begin{array}{l}\text { CINTICHEM to } \\
\text { Savannah River Project }\end{array}$ & $\begin{array}{l}\text { NC line } \\
\mathrm{I}-77 \\
\mathrm{I}-20\end{array}$ & $\begin{array}{l}I-77 \mathrm{~S} \\
\mathrm{I}-20 \mathrm{~W} \\
\text { SC-19 S }\end{array}$ & $\begin{array}{l}\text { I }-20 \\
\text { SC-19 } \\
\text { SRP }\end{array}$ & 1991 \\
\hline & $\begin{array}{l}\text { University of Missouri } \\
\text { to Savannah River } \\
\text { Project }\end{array}$ & $\begin{array}{l}\text { NC line } \\
\text { I-26 } \\
\text { SC-121 }\end{array}$ & $\begin{array}{l}I-26 \mathrm{~S} \\
\text { SC-121 S } \\
\text { SC-19S }\end{array}$ & $\begin{array}{l}\text { SC-121 } \\
\text { SC-19 } \\
\text { SRP }\end{array}$ & 1987 \\
\hline & $\begin{array}{l}\text { University of Virginia } \\
\text { to Savannah River } \\
\text { Project }\end{array}$ & & (same as abov & & $1987 / 88$ \\
\hline & $\begin{array}{l}\text { NIST to Savannah River } \\
\text { Project }\end{array}$ & & (same as abov & & 1987 \\
\hline & $\begin{array}{l}\text { Port of Savannah to } \\
\text { Savannah River Project }\end{array}$ & $\begin{array}{l}\text { GA line } \\
\text { I-95 } \\
\text { I-26 } \\
\text { US-301 } \\
\text { SC-70 }\end{array}$ & $\begin{array}{l}\text { I-95 N } \\
\text { I-26 W } \\
\text { US-301 W } \\
\text { SC-70 W } \\
\text { SC-64 W }\end{array}$ & $\begin{array}{l}\text { I-26 } \\
\text { US-301 } \\
\text { SC-70 } \\
\text { SC-64 } \\
\text { SRP }\end{array}$ & $1987 / 88$ \\
\hline & $\begin{array}{l}\text { Babcock \& Wilcox, } \\
\text { Lynchburg, to Oconee } \\
\text { Station }\end{array}$ & $\begin{array}{l}\text { NC line } \\
\text { I-26 } \\
\text { I-85 } \\
\text { SC-153 } \\
\text { US-123 }\end{array}$ & $\begin{array}{l}\text { I-26 S } \\
\text { I-85 SW } \\
\text { SC-153 N } \\
\text { US-123 SW } \\
\text { SC-130 N }\end{array}$ & $\begin{array}{l}\text { I-85 } \\
\text { SC-153 } \\
\text { US-123 } \\
\text { SC-130 } \\
\text { Oconee } \\
\text { Station }\end{array}$ & 1987 \\
\hline
\end{tabular}


Table 4.1 (Continued)

\begin{tabular}{|c|c|c|c|c|c|}
\hline \multirow[b]{2}{*}{ State } & \multirow[b]{2}{*}{ Shipment } & \multicolumn{3}{|c|}{ Highway Shipment Route Segment } & \multirow{2}{*}{$\begin{array}{l}\text { Shipments } \\
\text { Completed }\end{array}$} \\
\hline & & From: & Route & To: & \\
\hline $\begin{array}{l}\text { SC } \\
\text { (Cont.) }\end{array}$ & $\begin{array}{l}\text { Virgil C. Summer } \\
\text { Nuclear Generating Station } \\
\text { to Alexandria Bay }\end{array}$ & $\begin{array}{l}\text { Summer } \\
\text { SC-215 } \\
\text { SC-213 } \\
\text { SC-34 }\end{array}$ & $\begin{array}{l}\text { SC-215 N } \\
\text { SC-213 E } \\
\text { SC-34 E/N } \\
I-77 N\end{array}$ & $\begin{array}{l}\mathrm{SC}-213 \\
\mathrm{SC}-34 \\
\mathrm{I}-77 \\
\text { NC line }\end{array}$ & 1990 \\
\hline \multirow[t]{5}{*}{ TN } & $\begin{array}{l}\text { University of Michigan } \\
\text { to Savannah River } \\
\text { Project }\end{array}$ & $\begin{array}{l}\text { KY line } \\
I-75 \\
I-640\end{array}$ & $\begin{array}{l}I-75 S \\
I-640 \mathrm{E} \\
I-40 \mathrm{E}\end{array}$ & $\begin{array}{l}\text { I-640 } \\
\text { I-40 } \\
\text { NC line }\end{array}$ & 1987 \\
\hline & $\begin{array}{l}\text { University of Missouri } \\
\text { to Savannah River } \\
\text { Project }\end{array}$ & $\begin{array}{l}\text { KY line } \\
I-24 \\
I-65 \\
I-40 \\
I-640\end{array}$ & $\begin{array}{l}I-24 S \\
I-65 S \\
I-40 E \\
I-640 E \\
I-40 E\end{array}$ & $\begin{array}{l}I-65 \\
I-40 \\
I-640 \\
I-40 \\
\text { NC line }\end{array}$ & 1987 \\
\hline & $\begin{array}{l}\text { CINTICHEM to Savannah } \\
\text { River Project }\end{array}$ & $\begin{array}{l}\text { VA line } \\
I-81\end{array}$ & $\begin{array}{l}I-81 S \\
I-40 S\end{array}$ & $\begin{array}{l}1-40 \\
\text { NC line }\end{array}$ & $1987 / 91$ \\
\hline & $\begin{array}{l}\text { Babcock \& Wilcox, } \\
\text { Lynchburg, to Quad } \\
\text { Cities }\end{array}$ & $\begin{array}{l}\text { VA line } \\
\text { I-81 } \\
\text { I }-40\end{array}$ & $\begin{array}{l}\mathrm{I}-81 \mathrm{~S} \\
\mathrm{I}-40 \mathrm{~W} \\
\mathrm{I}-75 \mathrm{~N}\end{array}$ & $\begin{array}{l}\mathrm{I}-40 \\
\mathrm{I}-75 \\
\mathrm{KY} \text { line }\end{array}$ & 1987 \\
\hline & $\begin{array}{l}\text { Arkansas One to } \\
\text { Portsmouth, Virginia } \\
\text { Marine Terminal }\end{array}$ & $\begin{array}{l}\text { AR Line } \\
I-40 \\
I-240 \\
I-40\end{array}$ & $\begin{array}{l}I-40 \mathrm{E} \\
\mathrm{I}-240 \mathrm{SE} \\
\mathrm{I}-40 \mathrm{E} \\
I-81 \mathrm{~N}\end{array}$ & $\begin{array}{l}I-240 \\
\text { I-40 } \\
\text { I-81 } \\
\text { VA Line }\end{array}$ & 1991 \\
\hline \multirow[t]{7}{*}{ UT } & $\begin{array}{l}\text { University of Missouri } \\
\text { to Idaho National } \\
\text { Engineering Laboratory }\end{array}$ & $\begin{array}{l}\text { WY line } \\
\text { I-80 } \\
I-84\end{array}$ & $\begin{array}{l}\mathrm{I}-80 \mathrm{~W} \\
\mathrm{I}-84 \mathrm{~N} \\
\mathrm{I}-15 \mathrm{~N}\end{array}$ & $\begin{array}{l}\text { I-84 } \\
\text { I-15 } \\
\text { ID line }\end{array}$ & 1989 \\
\hline & $\begin{array}{l}\text { General Atomics to } \\
\text { Idaho National } \\
\text { Engineering Laboratory }\end{array}$ & AZ line & I $-15 \mathrm{~N}$ & ID line & 1987 \\
\hline & $\begin{array}{l}\text { Michigan State } \\
\text { University to Idaho } \\
\text { National Engineering } \\
\text { Laboratory }\end{array}$ & $\begin{array}{l}\text { WY line } \\
\mathrm{I}-80 \\
\mathrm{I}-84\end{array}$ & $\begin{array}{l}\mathrm{I}-80 \mathrm{~W} \\
\mathrm{I}-84 \mathrm{~N} \\
\mathrm{I}-15 \mathrm{~N}\end{array}$ & $\begin{array}{l}\mathrm{I}-84 \\
\mathrm{I}-15 \\
\text { ID line }\end{array}$ & 1989 \\
\hline & $\begin{array}{l}\text { Fort St. Vrain to Idaho } \\
\text { National Engineering } \\
\text { Laboratory }\end{array}$ & & (same as abc & & 1991 \\
\hline & $\begin{array}{l}\text { University of Calif. } \\
\text { Berkeley to Idaho } \\
\text { National Engineering } \\
\text { Laboratory }\end{array}$ & $\begin{array}{l}\text { NV line } \\
\mathrm{I}-80 \\
\mathrm{I}-215\end{array}$ & $\begin{array}{l}\mathrm{I}-80 \mathrm{E} \\
\mathrm{I}-215 \mathrm{~N} \\
\mathrm{I}-15 \mathrm{~N}\end{array}$ & $\begin{array}{l}\mathrm{I}-215 \\
\mathrm{I}-15 \\
\text { ID line }\end{array}$ & 1989 \\
\hline & $\begin{array}{l}\text { Portsmouth, Virginia, } \\
\text { Marine Terminal to } \\
\text { GE/Vallecitos }\end{array}$ & WY line & $\mathrm{I}-80 \mathrm{~W}$ & NV line & 1988 \\
\hline & $\begin{array}{l}\text { Babcock \& Wilcox, } \\
\text { Lynchburg, to } \\
\text { GE/Vallecitos }\end{array}$ & & \multicolumn{2}{|c|}{ (same as above route) } & 1987 \\
\hline
\end{tabular}


Table 4.1 (Continued)

\begin{tabular}{|c|c|c|c|c|c|}
\hline \multirow[b]{2}{*}{ State } & \multirow[b]{2}{*}{ Shipment } & \multicolumn{3}{|c|}{ Highway Shipment Route Segment } & \multirow{2}{*}{$\begin{array}{l}\text { Shipments } \\
\text { Completed }\end{array}$} \\
\hline & & From: & Route & To: & \\
\hline \multirow[t]{9}{*}{$\begin{array}{l}\text { VA } \\
\text { (Cont.) }\end{array}$} & $\begin{array}{l}\text { Oconee to Babcock \& } \\
\text { Wilcox, Lynchburg, }\end{array}$ & $\begin{array}{l}\text { NC line } \\
\text { I-77 } \\
\text { I-81 } \\
\text { I-581 } \\
\text { US-460 }\end{array}$ & $\begin{array}{l}\text { I-77 N } \\
\text { I-81 N } \\
\text { I-581 E } \\
\text { US-460 E } \\
\text { Mt. Athos Rd. E }\end{array}$ & $\begin{array}{l}\text { I-81 } \\
\text { I-581 } \\
\text { US-460 } \\
\text { Mt. Athos } \\
\text { Road } \\
\text { B\&W }\end{array}$ & $1988 / 89$ \\
\hline & $\begin{array}{l}\text { Newport News Terminal } \\
\text { to Savannah River } \\
\text { Project }\end{array}$ & $\begin{array}{l}\text { Term. } \\
\text { US-60 } \\
\text { US-17 } \\
\text { VA-32 } \\
\text { US-58 }\end{array}$ & $\begin{array}{l}\text { US-60 N } \\
\text { US-17 S } \\
\text { VA-32 S } \\
\text { US-58 W } \\
\text { I-95 S }\end{array}$ & $\begin{array}{l}\text { US-17 } \\
\text { VA-32 } \\
\text { US-58 } \\
\text { I-95 } \\
\text { NC line }\end{array}$ & 1989 \\
\hline & $\begin{array}{l}\text { Portsmouth, Virginia } \\
\text { Marine Terminal to } \\
\text { Savannah River Project }\end{array}$ & $\begin{array}{l}\text { Portsmouth } \\
\text { US-58 } \\
\text { US-17 } \\
\text { I-264 } \\
\text { US-58 }\end{array}$ & $\begin{array}{l}\text { US-58 W } \\
\text { US-17 S } \\
\text { I-264 W } \\
\text { US-58 W } \\
\text { I-95S }\end{array}$ & $\begin{array}{l}\text { US-17 } \\
\text { I-264 } \\
\text { US-58 } \\
\text { I-95 } \\
\text { NC line }\end{array}$ & $1987 / 88$ \\
\hline & $\begin{array}{l}\text { CINTICHEM to Savannah } \\
\text { River Project }\end{array}$ & WV line & $\mathrm{I}-81 \mathrm{~S}$ & TN line & 1987 \\
\hline & $\begin{array}{l}\text { CINTICHEM to } \\
\text { Savannah River Project }\end{array}$ & $\begin{array}{l}\text { WV line } \\
\text { I-81 }\end{array}$ & $\begin{array}{l}\mathrm{I}-81 \mathrm{~S} / \mathrm{W} \\
\mathrm{I}-77 \mathrm{~S}\end{array}$ & $\begin{array}{l}\text { I-77 } \\
\text { NC line }\end{array}$ & 1991 \\
\hline & $\begin{array}{l}\text { University of Virginia } \\
\text { to Savannah River } \\
\text { Project }\end{array}$ & $\begin{array}{l}\text { UVA } \\
\text { Alderman Rd. } \\
\text { US-250 } \\
\text { US-29 } \\
\text { I-64 } \\
\text { I-81 }\end{array}$ & $\begin{array}{l}\text { Alderman Rd. N } \\
\text { US-250 W } \\
\text { US-29 S } \\
\text { I-64 W } \\
\text { I-81 S } \\
\text { I-77 S }\end{array}$ & $\begin{array}{l}\text { US-250 } \\
\text { US-29 } \\
\text { I-64 } \\
\text { I-81 } \\
\text { I-77 } \\
\text { NC line }\end{array}$ & $1987 / 88$ \\
\hline & $\begin{array}{l}\text { Norfolk Int'1 Term. to } \\
\text { Savannah River Project }\end{array}$ & $\begin{array}{l}\text { Term. } \\
\text { Port roads } \\
\text { Term. Blvd } \\
\text { I-564 } \\
\text { I-64 } \\
\text { US-58 }\end{array}$ & $\begin{array}{l}\text { Port roads E } \\
\text { Term. Blvd. E } \\
\text { I-564 S } \\
\text { I-64 S } \\
\text { US-58 W } \\
\text { I-95 S }\end{array}$ & $\begin{array}{l}\text { Term. } \\
\text { Blvd. } \\
\text { I-564 } \\
\text { I-64 } \\
\text { US-58 } \\
\text { I-95 } \\
\text { NC line }\end{array}$ & 1987 \\
\hline & $\begin{array}{l}\text { Alexandria Bay to } \\
\text { Savannah River Project }\end{array}$ & $\begin{array}{l}\text { MD line } \\
I-81\end{array}$ & $\begin{array}{l}\mathrm{I}-81 \mathrm{~S} \\
\mathrm{I}-77 \mathrm{~S}\end{array}$ & $\begin{array}{l}\text { I-77 } \\
\text { NC line }\end{array}$ & 1988 \\
\hline & $\begin{array}{l}\text { Portsmouth, Virginia, } \\
\text { Marine Terminal to } \\
\text { GE/Vallecitos }\end{array}$ & $\begin{array}{l}\text { Ports. Marine } \\
\text { Term } \\
\text { US-58 } \\
\text { VA-17 } \\
\text { I-264 } \\
\text { US-460 } \\
\text { VA-32 } \\
\text { US-258 } \\
\text { I-64 } \\
\text { I-295 } \\
\text { I-64 }\end{array}$ & $\begin{array}{l}\text { US-58 S } \\
\text { VA-17 S } \\
\text { I-264 S } \\
\text { US-460 W } \\
\text { VA-32 N } \\
\text { US-258 N } \\
\text { I-64 W } \\
\text { I-295 W } \\
\text { I-64 W } \\
\text { I-81 N }\end{array}$ & $\begin{array}{l}\text { VA-17 } \\
\text { I-264 } \\
\text { US-460 } \\
\text { VA-32 } \\
\text { US-258 } \\
\text { I-64 } \\
\text { I-295 } \\
\text { I-64 } \\
\text { I-81 } \\
\text { WV line }\end{array}$ & 1988 \\
\hline
\end{tabular}


Table 4.1 (Continued)

\begin{tabular}{|c|c|c|c|c|c|}
\hline \multirow[b]{2}{*}{ State } & \multirow[b]{2}{*}{ Shipment } & \multicolumn{3}{|c|}{ Highway Shipment Route Segment } & \multirow{2}{*}{$\begin{array}{l}\text { Shipments } \\
\text { Completed }\end{array}$} \\
\hline & & From: & Route & To: & \\
\hline \multirow[t]{8}{*}{$\begin{array}{l}\text { VA } \\
\text { (Cont.) }\end{array}$} & $\begin{array}{l}\text { Dresden Reactor to } \\
\text { Portsmouth, Virginia } \\
\text { Marine Terminal }\end{array}$ & & \multicolumn{2}{|c|}{ (reverse of above route) } & 1987 \\
\hline & $\begin{array}{l}\text { Arkansas One to } \\
\text { Portsmouth Marine } \\
\text { Terminal }\end{array}$ & $\begin{array}{l}\text { TN Line } \\
\text { I-84 } \\
\text { I-81/I-64 } \\
\text { I-64 } \\
\text { I-295 } \\
\text { I-64 } \\
\text { US-258 } \\
\text { VA-82 } \\
\text { US-460 } \\
\text { I-264 } \\
\text { VA-17 }\end{array}$ & $\begin{array}{l}\mathrm{I}-84 \mathrm{~N} \\
\mathrm{I}-81 / \mathrm{I}-64 \mathrm{~N} / \mathrm{E} \\
\mathrm{I}-64 \mathrm{E} \\
\mathrm{I}-295 \mathrm{E} \\
\mathrm{I}-64 \mathrm{E} \\
\text { US-258 S } \\
\text { VA-32 E } \\
\text { US-460 E } \\
\mathrm{I}-264 \mathrm{E} \\
\text { VA-17N } \\
\text { US-58 }\end{array}$ & $\begin{array}{l}\text { I-81/I-64 } \\
\text { I-64 } \\
\text { I-295 } \\
\text { I-64 } \\
\text { US-258 } \\
\text { VA-82 } \\
\text { US-460 } \\
\text { I-264 } \\
\text { VA-17 } \\
\text { US-58 } \\
\text { Portsmouth } \\
\text { Marine Terminal }\end{array}$ & 1991 \\
\hline & $\begin{array}{l}\text { NIST to Savannah River } \\
\text { Project }\end{array}$ & $\begin{array}{l}\text { WV line } \\
\text { I-81 }\end{array}$ & $\begin{array}{l}\mathrm{I}-81 \mathrm{~S} \\
\mathrm{I}-77 \mathrm{~S}\end{array}$ & $\begin{array}{l}\mathrm{I}-77 \\
\text { NC line }\end{array}$ & 1987 \\
\hline & $\begin{array}{l}\text { Babcock \& Wilcox, } \\
\text { Lynchburg, to Oconee } \\
\text { Station }\end{array}$ & $\begin{array}{l}\text { Site W } \\
\text { Mt. Athos Rd. } \\
\text { US-460 } \\
\text { I-581 W } \\
\text { I-81 }\end{array}$ & $\begin{array}{l}\text { Mt. Athos Rd. W } \\
\text { US-460 W } \\
\text { I-581 W } \\
\text { I-81 W } \\
\text { I-77 S }\end{array}$ & $\begin{array}{l}\text { US-460 } \\
\text { I-581 } \\
\text { I-81 } \\
\text { I-77 } \\
\text { NC line }\end{array}$ & 1987 \\
\hline & $\begin{array}{l}\text { Babcock \& Wilcox, } \\
\text { Lynchburg, to } \\
\text { GE/Vallecitos }\end{array}$ & $\begin{array}{l}\text { Site } \\
\text { VA-726 } \\
\\
\text { US-460 } \\
\text { VA-220 Alt. } \\
\text { US-11 }\end{array}$ & $\begin{array}{l}\text { VA-726 S } \\
\text { US-460 W } \\
\\
\text { VA-220 Alt. N } \\
\text { US-11 N } \\
\text { I-81 N }\end{array}$ & $\begin{array}{l}\text { US-460 } \\
\text { VA-220 } \\
\text { Alt. } \\
\text { US-11 } \\
\text { I-81 } \\
\text { WV line }\end{array}$ & 1987 \\
\hline & $\begin{array}{l}\text { Dresden Reactor to } \\
\text { Babcock \& Wilcox, } \\
\text { Lynchburg }\end{array}$ & $\begin{array}{l}\text { WV line } \\
\text { I-81 } \\
\text { US-11 } \\
\text { VA-220 Alt. } \\
\text { US-460 }\end{array}$ & $\begin{array}{l}\text { I-81 S } \\
\text { US-11 S } \\
\text { VA-220 Alt. S } \\
\text { US-460 E } \\
\text { Mt. Athos Rd. E }\end{array}$ & $\begin{array}{l}\text { US-11 } \\
\text { VA-220 } \\
\text { Alt. } \\
\text { US-460 } \\
\text { Mt. Athos } \\
\text { Road } \\
\text { B\&W }\end{array}$ & 1987 \\
\hline & $\begin{array}{l}\text { Babcock \& Wilcox, } \\
\text { Lynchburg, to Quad } \\
\text { Cities }\end{array}$ & $\begin{array}{l}\text { Site } \\
\text { Mt. Athos Rd. } \\
\text { US-460 } \\
\text { I-581 }\end{array}$ & $\begin{array}{l}\text { Mt. Athos Rd. W } \\
\text { US-460 W } \\
\text { I-581 W } \\
\text { I-81 S }\end{array}$ & $\begin{array}{l}\text { US-460 } \\
\text { I-581 } \\
\text { I- } 81 \\
\text { TN line }\end{array}$ & 1987 \\
\hline & $\begin{array}{l}\text { Summer to } \\
\text { Alexandria Bay }\end{array}$ & $\begin{array}{l}\text { NC line } \\
\text { I-77 }\end{array}$ & $\begin{array}{l}\mathrm{I}-77 \mathrm{~N} \\
\mathrm{I}-81\end{array}$ & $\begin{array}{l}\text { I-81 } \\
\text { WV line }\end{array}$ & 1990 \\
\hline \multirow[t]{2}{*}{ WV } & $\begin{array}{l}\text { Alexandria Bay to } \\
\text { Savannah River Project }\end{array}$ & MD line & $\mathrm{I}-81 \mathrm{~S}$ & VA line & 1988 \\
\hline & $\begin{array}{l}\text { CINTICHEM to Savannah } \\
\text { River Project }\end{array}$ & MD line & $\mathrm{I}-81 \mathrm{~S}$ & VA line & $1987 / 91$ \\
\hline
\end{tabular}


Table 4.1 (Continued)

\begin{tabular}{|c|c|c|c|c|c|}
\hline \multirow[b]{2}{*}{ State } & \multirow[b]{2}{*}{ Shipment } & \multicolumn{3}{|c|}{ Highway Shipment Route Segment } & \multirow{2}{*}{$\begin{array}{l}\text { Shipments } \\
\text { Completed }\end{array}$} \\
\hline & & From: & Route & To: & \\
\hline \multirow[t]{7}{*}{$\begin{array}{l}\text { WV } \\
\text { (Cont.) }\end{array}$} & $\begin{array}{l}\text { Dresden Reactor to } \\
\text { Portsmouth, Virginia, } \\
\text { Marine Terminal }\end{array}$ & $\begin{array}{l}\text { OH line } \\
\text { I-470 } \\
\text { PA line } \\
\text { I-79 } \\
\text { MD line }\end{array}$ & $\begin{array}{l}\mathrm{I}-470 \mathrm{E} \\
\mathrm{I}-70 \mathrm{E} \\
\mathrm{I}-79 \mathrm{~S} \\
\mathrm{US}-48 \mathrm{E} \\
\mathrm{I}-81 \mathrm{~S}\end{array}$ & $\begin{array}{l}\text { I-70 } \\
\text { PA Line } \\
\text { US-48 } \\
\text { MD line } \\
\text { VA line }\end{array}$ & 1987 \\
\hline & $\begin{array}{l}\text { Dresden Reactor to } \\
\text { Babcock \& Wilcox, } \\
\text { Lynchburg }\end{array}$ & & \multicolumn{2}{|c|}{ (same as above route) } & 1987 \\
\hline & $\begin{array}{l}\text { Portsmouth, Virginia, } \\
\text { Marine Terminal to } \\
\text { GE/Vallecitos }\end{array}$ & & \multicolumn{2}{|c|}{ (reverse of above route) } & 1988 \\
\hline & $\begin{array}{l}\text { Callaway to } \\
\text { Alexandria Bay }\end{array}$ & $\begin{array}{l}\text { OH line } \\
\mathrm{I}-470\end{array}$ & $\begin{array}{l}I-470 E \\
I-70 E\end{array}$ & $\begin{array}{l}\text { I-70 } \\
\text { PA line }\end{array}$ & 1991 \\
\hline & $\begin{array}{l}\text { Babcock \& Wilcox, } \\
\text { Lynchburg, to } \\
\text { GE/Vallecitos }\end{array}$ & & \multicolumn{2}{|c|}{ (reverse of above route) } & 1987 \\
\hline & $\begin{array}{l}\text { NIST to Savannah River } \\
\text { Project }\end{array}$ & MD line & $\mathrm{I}-81 \mathrm{~S}$ & VA line & 1987 \\
\hline & $\begin{array}{l}\text { Summer to } \\
\text { Alexandria Bay }\end{array}$ & & \multicolumn{2}{|c|}{ (reverse of above) } & 1990 \\
\hline \multirow[t]{6}{*}{ WY } & $\begin{array}{l}\text { University of Missouri } \\
\text { to Idaho National } \\
\text { Engineerirg Laboratory }\end{array}$ & NE line & $\mathrm{I}-80 \mathrm{~W}$ & UT line & 1989 \\
\hline & $\begin{array}{l}\text { Michigan State } \\
\text { University to Idaho } \\
\text { National Engineering } \\
\text { Laboratory }\end{array}$ & & \multicolumn{2}{|c|}{ (same as above route) } & 1989 \\
\hline & $\begin{array}{l}\text { Babcock \& Wilcox, } \\
\text { Lynchburg, to } \\
\text { GE/Vallecitos }\end{array}$ & & \multicolumn{2}{|c|}{ (same as above route) } & 1987 \\
\hline & $\begin{array}{l}\text { Fort St. Vrain to } \\
\text { Idaho National Engineering } \\
\text { Laboratory }\end{array}$ & $\begin{array}{l}\text { CO line } \\
1-25\end{array}$ & $\begin{array}{l}\mathrm{I}-25 \mathrm{~N} \\
\mathrm{I}-80 \mathrm{~W}\end{array}$ & $\begin{array}{l}\text { I-80 } \\
\text { UT line }\end{array}$ & 1991 \\
\hline & $\begin{array}{l}\text { Portsmouth, Virginia, } \\
\text { Marine Terminal to } \\
\text { GE/Vallecitos }\end{array}$ & & \multicolumn{2}{|c|}{ (same as above route) } & 1988 \\
\hline & $\begin{array}{l}\text { Michigan State } \\
\text { University to Denver } \\
\text { Federal Center }\end{array}$ & $\begin{array}{l}\text { NE line } \\
\mathrm{I}-80\end{array}$ & $\begin{array}{l}I-80 \mathrm{~W} \\
\mathrm{I}-25 \mathrm{~S}\end{array}$ & $\begin{array}{l}\text { I-25 } \\
\text { CO line }\end{array}$ & 1989 \\
\hline
\end{tabular}


Table 4.1 (Continued)

\begin{tabular}{|c|c|c|c|c|c|}
\hline \multirow[b]{2}{*}{ State } & \multirow[b]{2}{*}{ Shipment } & \multicolumn{3}{|c|}{ Railway Shipment Route Segment } & \multirow{2}{*}{$\begin{array}{l}\text { Shipments } \\
\text { Completed }\end{array}$} \\
\hline & & From: & Route & To: & \\
\hline \multirow[t]{3}{*}{ IL } & \multirow[t]{2}{*}{$\begin{array}{l}\text { Cooper Reactor } \\
\text { to GE/Morris }\end{array}$} & IA line & $\begin{array}{l}\text { Burlington } \\
\text { Northern }\end{array}$ & EOLA & $1987 / 88 / 89$ \\
\hline & & EOLA & $\begin{array}{l}\text { Elgin, } \\
\text { Joliet, } \\
\text { Eastern }\end{array}$ & GE/Morris & \\
\hline & $\begin{array}{l}\text { Monticello to } \\
\text { GE/Morris }\end{array}$ & & \multicolumn{2}{|l|}{ (Same as above route) } & 1987 \\
\hline IA & $\begin{array}{l}\text { Cooper Reactor to } \\
\text { GE/Morris }\end{array}$ & NE line & $\begin{array}{l}\text { Burlington } \\
\text { Northern }\end{array}$ & IL line & $1987 / 88 / 89$ \\
\hline $\mathrm{MN}$ & $\begin{array}{l}\text { Monticello to } \\
\text { GE/Morris }\end{array}$ & Monticello & $\begin{array}{l}\text { Burlington } \\
\text { Northern }\end{array}$ & WI line & 1987 \\
\hline NE & $\begin{array}{l}\text { Cooper Reactor } \\
\text { to GE/Morris }\end{array}$ & Cooper & $\begin{array}{l}\text { Burlington } \\
\text { Northern }\end{array}$ & IA line & $1987 / 88 / 89$ \\
\hline \multirow[t]{3}{*}{$\mathrm{NC}$} & $\begin{array}{l}\text { Brunswick to } \\
\text { Shearon Harris }\end{array}$ & $\begin{array}{l}\text { Brunswick } \\
\text { Leland } \\
\text { Hamlet }\end{array}$ & $\begin{array}{l}\text { Military Ocean } \\
\text { Term. track } \\
\text { CSX } \\
\text { CSX }\end{array}$ & $\begin{array}{l}\text { Leland } \\
\text { Hamlet } \\
\text { Bonsal } \\
\text { (Shearon } \\
\text { Harris) }\end{array}$ & 1989 \\
\hline & $\begin{array}{l}\text { Robinson to } \\
\text { Shearon Harris }\end{array}$ & $\begin{array}{l}\text { SC line } \\
\text { Hamlet }\end{array}$ & $\begin{array}{l}\text { CSX } \\
\text { CSX }\end{array}$ & $\begin{array}{l}\text { Hamlet } \\
\text { Bonsal } \\
\text { (Shearon } \\
\text { Harris) }\end{array}$ & $1990 / 91$ \\
\hline & $\begin{array}{l}\text { Robinson to } \\
\text { Brunswick }\end{array}$ & SC line & $\begin{array}{l}\text { Seaboard } \\
\text { Coast Line (SCL) }\end{array}$ & Brunswick & $1979 / 80 / 81$ \\
\hline \multirow[t]{2}{*}{ SC } & $\begin{array}{l}\text { Robinson to } \\
\text { Shearon Harris }\end{array}$ & Robinson & $\operatorname{CSX}$ & NC line & $1990 / 91$ \\
\hline & $\begin{array}{l}\text { Robinson to } \\
\text { Brunswick }\end{array}$ & Robinson & $\mathrm{SCL}$ & NC line & $1979 / 80 / 81$ \\
\hline WI & $\begin{array}{l}\text { Monticello to } \\
\text { GE/Morris }\end{array}$ & MN line & $\begin{array}{l}\text { Burlington } \\
\text { Northern }\end{array}$ & IL line & 1987 \\
\hline
\end{tabular}


. 


\begin{tabular}{|c|c|}
\hline $\begin{array}{l}\text { U.S NUCLEAR REGULATOAY COMMISSION } \\
\text { BIBLIOGRAPHIC DATA SHEET } \\
\text { (See instructions on the reverse) }\end{array}$ & $\begin{array}{l}\text { 1. REPORT NUMBER } \\
\text { (Assigned by NRC, Add Vol } \\
\text { Supp, Rev, and Addendum Num- } \\
\text { bers, if any') }\end{array}$ \\
\hline \multirow{4}{*}{$\begin{array}{l}2 \text { TITLE AND SUBTITLE } \\
\text { Public Information Circular for Shipments of } \\
\text { Irradiated Reactor Fuel }\end{array}$} & NUREG-0725, Rev. 8 \\
\hline & 3 DATE REPORT PUBLISHED \\
\hline & \begin{tabular}{l|c} 
MONTH & YEAR \\
June & 1992
\end{tabular} \\
\hline & 4 FIN OR GRANT NUMBER \\
\hline \multirow[t]{2}{*}{5 AUTHOR(S) } & $\begin{array}{l}6 \text { TYPE OF REPORT } \\
\text { Regulatory }\end{array}$ \\
\hline & $\begin{array}{l}7 \text { PERIOD COVERED (Inclusive Dates) } \\
10 / 01 / 87 \text { to } 12 / 31 / 91\end{array}$ \\
\hline \multicolumn{2}{|c|}{$\begin{array}{l}8 \text { PERFORMING ORGANIZATION - NAME AND ADDRESS (If NRC, provide DIVision, Office or Region, US Nuclear Regulatory Commission, and } \\
\text { mailing address, if contractor, provide name and mailing address ) } \\
\text { Division of Safeguards and Transportation } \\
\text { Office of Nuclear Material Safety and Safeguards } \\
\text { U.S. Nuclear Regulatory Commission } \\
\text { Washington, DC } 20555\end{array}$} \\
\hline
\end{tabular}

10 SUPPLEMENTARY NOTES

Updates expected to be issued periodically

11 ABSTAACT (200 words or less)

This circular has been prepared to provide information on the shipment of irradiated reactor fuel (spent fuel) subject to regulation by the Nuclear Regulatory Commission (NRC), and to meet the requirements of Public Law 96-295. The report provides a brief description of NRC authority for certain aspects of transporting spent fuel. It provides descriptive statistics on spent fuel shipments regulated by the NRC from 1979 to 1991 . It also lists detailed highway and railway segments used within each state from October 1, 1987 through December 31, 1991. 